\title{
Distributional and Peer-induced Fairness in Supply Chain Contract Design
}

\author{
Teck-Hua Ho, Xuanming Su, and Yaozhong Wu *
}

January 27, 2013

Members of a supply chain often make profit comparisons. A retailer exhibits peer-induced fairness concerns when his own profit is behind that of a peer retailer interacting with the same supplier. In addition, a retailer exhibits distributional fairness concerns when his supplier's share of total profit is disproportionately larger than his own. While existing research focuses exclusively on distributional fairness concerns, this paper investigates how both types of fairness concerns might interact and influence economic outcomes in a supply chain. We consider a setting where a supplier sells an identical product through 2 independent retailers, each serving his own market. The supplier sequentially offers each retailer a linear wholesale price contract, and each retailer must choose his own retail price if he accepts the supplier's wholesale price offer. The second retailer observes a noisy signal of the first wholesale price offer and this information may influence his decisions. We show that: (i) the first wholesale price offer is lower than the standard wholesale price offer in the absence of fairness concerns, (ii) the second wholesale price is higher than the first wholesale price, and (iii) the second retailer makes a lower profit and has a lower share of the total supply chain profit than the first retailer. We run controlled experiments with subjects motivated by substantial monetary incentives and show that subject behaviors are consistent with the model predictions. Structural estimation on the data suggests that peer-induced fairness is more salient than distributional fairness.

Keywords: Distributional Fairness, Peer-induced Fairness, Supply Chain Contracting, Behavioral Operations Management, Behavioral Economics

*All authors contributed equally. The authors are listed in alpabetical order. Direct correspondence to any of the authors. Ho:hoteck@haas.berkeley.edu, Su:xuanming@wharton.upenn.edu, Wu:bizwyz@nus.edu.sg. We thank Jin Qi, Xing Zhang, and Li Xiao for their superb research assistance. 


\section{Introduction}

Fairness is a cornerstone in our daily social interactions. We all want to be treated fairly by our friends and colleagues. This paper studies two types of fairness concerns: distributional fairness, where people dislike unfavorable shares in a distribution of a total pie, and peer-induced fairness, where people dislike unfavorable treatment relative to a peer. Peo-

ple tend to penalize unfair behavior even at their own expense. For example, customers frequently respond to firms' price gouging practices by boycotting them, a phenomenon arising from customers' distributional fairness concerns (Fehr and Schmidt, 1999; Bolton and Ockenfels, 2000; Charness and Rabin, 2002). Also, customers are averse to being behind other customers in terms of economic outcomes because they have peer-induced fairness concerns (Ho and $\mathrm{Su}, 2009$ ).

Fairness matters in business-to-business transactions too (Kahneman et. al, 1986, Anderson and Weitz, 1992). A retailer who feels that his supplier prices unfairly may retaliate by raising retail price in order to reduce the supplier's share of the total channel surplus. Likewise, a retailer who has a good guess of what a peer retailer's wholesale price offer may compare his profit with that of the peer retailer and adjust his retail price in order not to be behind. To the best of our knowledge, there is no research investigating the interaction between the two kinds of fairness in the design of supply chain contracts.

This paper considers a 1-supplier and 2-retailers supply chain and investigates, both theoretically and experimentally, the role of distributional and peer-induced fairness in supply chain contract design. We first analyze how distributional fairness affects both wholesale and retail prices between a supplier and a retailer. As shown extensively in the experimental and behavioral economics literature, a seller who demands a higher proportion of a fixed pie by charging a take-or-leave-it ultimatum price offer is frequently rejected and penalized by a buyer. While the standard ultimatum game may capture a business-to-customer retail market well, the game fails to capture the reality of the strategic interaction between members of a supply chain. This is because even in the simplest possible 1-supplier and 1-retailer supply chain, the size of the pie is not exogenously fixed but determined by the retailer through his retail price decision. In this paper, we analyze how optimal wholesale and retail prices change when the retailer is 
allowed to have distributional fairness concerns.

We then extend the model by introducing peer-induced fairness in a 1-supplier and 2retailer supply chain where the supplier must determine his wholesale price offers to 2 retailers sequentially. First, the supplier offers a wholesale price contract to the first retailer. Then, the second retailer observes an imperfect signal of the first wholesale price offer. Finally, the supplier makes a wholesale price offer to the second retailer. In this setup, the second retailer's willingness to accept the contract may depend on what he thinks the first retailer received. As a result of peer-induced fairness concerns, the optimal wholesale and retail prices may change. The general model analyzes these changes and their accompanying implications on retailer's profitability and share of the total channel surplus.

Our general model predicts that distributional fairness results in a lower wholesale price offer by the supplier. In addition, the model predicts that the second retailer receives a higher wholesale price offer and receives a lower profit than the first retailer. One might expect the reverse result since the supplier may wish to allay the second retailer's peerinduced fairness concerns. However, we show the contrary. The supplier increases the wholesale price offer to the second retailer, because the latter must choose a retail price to balance the opposing forces of not being behind the supplier and not being behind the first retailer.

We conduct economic experiments with subjects motivated by substantial monetary incentives and show that subjects' behaviors are consistent with the model's main predictions. In addition, we structurally estimate our model with the experimental data and find that both distributional and peer-induced fairness are important in describing subjects' behaviors. In addition, peer-induced fairness appears more salient than distributional fairness in determining subjects' behaviors.

There has been growing behavioral research in operations management in recent years (Loch and $\mathrm{Wu}$ 2007). Decision biases, such as reference dependence and loss aversion (Kahneman and Tversky 1979), have been extensively studied in the context of supply 
chain contracting (e.g., Lim and Ho 2007; Ho and Zhang 2008; Su 2008; Katok and Wu 2009; Ho et al. 2010, Kalkanci et al. 2011). While this stream of literature finds boundedly rational decision-makers fail to make the optimal decisions predicted by standard models, research on social preferences shows both positive effects of fairness and reciprocity on performance (Cui et al. 2007, Loch and Wu 2008, Wu et al. 2011) and negative effects of social comparison among peers in making inventory decisions (Avci et al. 2012). Distributional fairness between retailer and supplier has been shown, both theoretically and experimentally, to contribute significantly to coordination failures and efficiency loss in supply chains, in particular when supply chain members are not fully informed of other members' fairness concerns (Katok et al. 2012, Katok and Pavlov 2012, Pavlov and Katok 2012).

Most existing research, however, has not addressed behavioral issues beyond a simple supply chain dyad (for an exception, see Ho et al. 2010). This research investigates social preferences in a 1-supplier and 2-retailer supply chain. This paper distinguishes from the above behavioral studies by making three contributions:

1. This research is the first to theoretically investigate the interaction between distributional and peer-induced fairness in a 1-supplier and 2-retailer supply chain. Our theoretical result that the second retailer who has peer-induced concerns receives a higher wholesale price and a lower profit is new and surprising.

2. We test our general model in economic experiments with financially motivated subjects. Our experimental results support the model's main predictions. Specifically, we show that: (i) the first retailer's wholesale price offer is lower than the standard "no-fairness" benchmark, (ii) the second retailer's wholesale price offer is higher than that of the first retailer, and (iii) the second retailer makes a smaller profit and receives a lower share of the total pie than the first retailer.

3. We structurally estimate our model and show that both distributional and peerinduced fairness parameters are significant and important in describing actual behaviors. Since the standard model (without fairness) is nested as as special case, our approach can formally quantify the role of fairness in price contract design.

This paper is organized as follows. Section 2 describes the basic model, the model with distributional fairness, and the general model with both distributional and peer-induced 
fairness. We prove three propositions about wholesale price offers and retailer's profitability and formulate them into 3 testable hypotheses. Section 3 describes the experimental design and procedure. Section 4 reports summary statistics of the experimental data, provides statistical tests of the three hypotheses, and estimates the model structurally. Section 5 provides an in-depth interpretation of the estimated structural models. Section 6 discusses managerial implications of the results. Section 7 concludes and suggests future research directions.

\section{Model Formulation}

\section{$2.1 \quad$ Notations}

Let us begin with some notation. The general model will analyze a supply chain with three players: Supplier (denoted by $S$ ) and Retailers 1 and 2 (denoted as $R_{1}$ and $R_{2}$ respectively). The supplier sells an identical product through the two retailers. The retailers operate in two separate markets, and have the same but independent demand curve as: $d_{i}=a-p_{i}, i=1,2$. The supplier has a constant marginal cost $c$, where $0<c<a$. The supplier determines wholesale price offers in sequence, with $w_{1}$ (offer to $R_{1}$ ) preceding $w_{2}$ (offer to $R_{2}$ ). If a retailer $i$ accepts the supplier's wholesale price offer, he must set a retailer price $p_{i}$ accordingly in order to maximize his utility.

In the next 3 subsections, we consider 3 increasingly general versions of the model. We first study a basic model with no fairness concerns, followed by a model with only distributional fairness concerns, and then finally the full model with both distributional and peer-induced fairness concerns. We refer to them as Models I, II and III respectively.

\subsection{Model I: No Fairness}

In the basic model, we consider a supplier and a retailer $i$ dyad. Here, the profit function of retailer $i$ is given by $\pi_{i}\left(p_{i}\right)=d_{i} \cdot\left(p_{i}-w_{i}\right)=\left(a-p_{i}\right) \cdot\left(p_{i}-w_{i}\right)$. The supplier's profit is given by $\pi_{S, i}\left(w_{i}\right)=d_{i} \cdot\left(w_{i}-c\right)=\left(a-p_{i}\right) \cdot\left(w_{i}-c\right)$. Conditional on a wholesale

price offer $w_{i}$, retailer $i$ 's best response function is $p_{i}\left(w_{i}\right)=\frac{a+w_{i}}{2}$. Substituting this best response function into the supplier's profit function, we have $\pi_{S, i}\left(w_{i}\right)=\frac{a-w_{i}}{2} \cdot\left(w_{i}-c\right)$ 
which is maximized by choosing $w_{i}^{o}=\frac{a+c}{2}$. Retailer $i$ 's optimal retail price is then given by $p_{i}^{o}=\frac{a+w_{i}^{o}}{2}=\frac{3 a+c}{4}$. Furthermore, in equilibrium, retailer $i$ earns $\pi_{i}^{o}=\frac{(a-c)^{2}}{16}$, and the supplier earns $\pi_{S, i}^{o}=\frac{(a-c)^{2}}{8}$. Note that the supplier makes twice as much profit as the retailer in the basic model. That is, the supplier enjoys $\frac{2}{3}$ and the retailer enjoys $\frac{1}{3}$ of the total channel profit.

\subsection{Model II: Distributional Fairness}

We now extend the basic model to allow retailer $i$ to have distributional fairness concerns. Specifically, the retailer cares not only about his own profit, but also his profit relative to the supplier's profit. As a consequence, the retailer $i$ incurs a disutility of making less than the supplier. Retailer $i$ 's revised utility is modeled as follows

$$
u_{i}= \begin{cases}\pi_{i}-\delta \cdot \max \left\{\pi_{S, i}-\pi_{i}, 0\right\}, & \text { if Accept } \\ 0 & \text { if Reject }\end{cases}
$$

where $\delta \geq 0$ is the distributional fairness parameter. ${ }^{1}$ Note that when $\delta=0, u_{i}=\pi_{i}$ the model reduces back to the basic model.

Proposition 1 characterizes the optimal wholesale and retail prices at equilibrium:

Proposition 1. Conditional on a wholesale price offer $w_{i}$, the retailer $i$ 's best-response retail price is as follows:

$$
p_{i}= \begin{cases}\frac{a+w_{i}}{2}+\frac{\delta\left(w_{i}-c\right)}{2(1+\delta)}, & \text { if } w_{i} \geq \frac{a(1+\delta)+c(2+\delta)}{3+2 \delta} \\ 2 w_{i}-c, & \frac{a+2 c}{3} \leq w_{i}<\frac{a(1+\delta)+c(2+\delta)}{3+2 \delta} \\ \frac{a+w_{i}}{2}, & \text { if } w_{i}<\frac{a+2 c}{3}\end{cases}
$$

Applying backward induction, the supplier's optimal wholesale price is given by

$$
w_{i}^{*}= \begin{cases}\frac{a+c}{2}-\frac{\delta(a-c)}{2(1+2 \delta)}, & \text { if } \delta<\frac{1}{7} \\ \frac{a+2 c}{3}, & \text { otherwise }\end{cases}
$$

As a consequence, the optimal retail price is

$$
p_{i}^{*}= \begin{cases}\frac{3 a+c}{4}, & \text { if } \delta<\frac{1}{7} \\ \frac{2 a+c}{3}, & \text { otherwise }\end{cases}
$$

\footnotetext{
${ }^{1}$ Since the supplier makes more money in the basic model, the retailer is always behind the supplier in terms of profitability.
} 
Note that when distributional fairness parameter $\delta<\frac{1}{7}$, the optimal wholesale price is smaller than that of the basic model but the retail price remains unchanged at $\frac{3 a+c}{4}$ (see Cui et. al 2007 for a related model). As a consequence, the total channel surplus remains the same but the retailer now enjoys a higher share of the surplus when compared to the basic model. When $\delta \geq \frac{1}{7}$, both the wholesale and retail prices are smaller than those in the basic model. In this case, the total channel surplus becomes larger than that in the basic model.

The same prediction should carry through when the supplier is faced with two independent retailers (with an identical demand function) as long as the supplier's wholesale price offers are made simultaneously and no retailer observes any signal of other retailer's offer. This is so because the supplier will make an identical offer to both retailers and there will not be any peer-induced fairness between them. Hence $w_{1}^{*}=w_{2}^{*}=w_{i}^{*}$.

\subsection{Model III: Distributional and Peer-induced Fairness}

We now consider a supply chain with 1 supplier and 2 retailers. The order of events is as follows. First, the supplier offers Retailer 1 the wholesale price $w_{1}$, and Retailer 1 sets the retail price $p_{1}^{* *}\left(w_{1}\right)$ if he accepts. Next, Retailer 2 observes a noisy signal $z=w_{1}+\epsilon$ of Retailer 1's wholesale price offer. The supplier observes signal $z$ as well. Finally, contingent on the signal $z$, the supplier offers Retailer 2 the wholesale price $w_{2}^{* *}(z)$, and Retailer 2 sets the retail price $p_{2}^{* *}\left(w_{2}, z\right)$ if he accepts.

In our model, Retailer 2 possesses noisy rational expectations. That is, Retailer 2 has the correct expectation of the true (but unobserved) wholesale price offer to Retailer 1 , but has some uncertainty over his belief. ${ }^{2}$ Specifically, Retailer 2 has the normal prior belief $w \sim \mathcal{N}\left(\mu, \sigma^{2}\right)$ over the first wholesale price, where the mean $\mu=w_{1}$ is correct and the standard deviation $\sigma$ reflects the level of uncertainty in Retailer 2's prior belief. Let $f$ denote the probability density function of this prior.

\footnotetext{
${ }^{2}$ Our model reflects common practice where price decisions are typically kept confidential, and unobserved prices may be inferred imperfectly from other observables. In the less common scenario where prices are fully revealed, our model can be applied by taking the limit $\sigma \rightarrow 0$.
} 
After observing the signal $z$, Retailer 2 forms a posterior belief of $w_{1}$. Consistent with our experiment reported below, we assume that $\epsilon$ is discrete and uniformly distributed over $[-b,+b], \quad b>0$. Therefore, possible values of $w_{1}$ are given by $\tilde{w} \in[z-b, z+b]$, and Retailer 2's belief is updated according to Bayes theorem as follows:

$$
p(\tilde{w}=z+\kappa)=\frac{f(z+\kappa)}{\sum_{\ell=-b}^{\ell=b} f(z+\ell)}, \kappa=-b,-b+1, \ldots, b-1, b .
$$

Based on the posterior beliefs, Retailer 2 can make inferences about Retailer 1's profits to determine whether he is ahead or behind. Let $\hat{p}(z)$ be the inferred probability that Retailer 1 has accepted the supplier's wholesale price offer, and let $\hat{\pi}_{1}(z)$ be the inferred expected profit of Retailer 1 conditional on acceptance. Retailer 2 infers $\hat{p}(z)$ and $\hat{\pi}_{1}(z)$ as follows: for each possible offer $\tilde{w}=z-\epsilon, \epsilon \in[-b, b]$, Retailer 1 accepts $\tilde{w}$ if $u_{1}(\tilde{w})>0$ (here $u_{1}$ is the utility of Retailer 1's best response to $\tilde{w}$ assuming acceptance). Let $\hat{w}=z+\hat{\kappa}$ denote the highest wholesale price offer acceptable to Retailer 1 . Thus, the probability that Retailer 1 has accepted the supplier's offer and become a peer is given by the summation of all posterior probabilities where $\tilde{w} \leq \hat{w}$ :

$$
\hat{p}(z)=\sum_{\kappa=-b}^{\hat{\kappa}} p(z+\kappa)=\frac{\sum_{\kappa \leq \hat{\kappa}} f(z+\kappa)}{\sum_{\ell=-b}^{\ell=b} f(z+\ell)} .
$$

Conditional on acceptance, the normalized probability of the acceptable offers (i.e., $\tilde{w} \leq$ $\hat{w}$ or $\kappa \leq \hat{\kappa})$ is

$$
q(\tilde{w}=z+\kappa)=\frac{p(z+\kappa)}{\hat{p}(z)}, \kappa=-b,-b+1, \ldots, \hat{\kappa} .
$$

Let $\pi_{1}(\tilde{w})$ be the Retailer 1's equilibrium profit when the wholesale price offer is $\tilde{w}$, then the expected profit according to Retailer 2's belief is given by

$$
\hat{\pi}_{1}(z)=\sum_{\kappa=-b}^{\hat{\kappa}} q(z+k) \pi_{1}(z+k) .
$$

This inferred expected profit of Retailer 1 then becomes a reference point for social comparison by Retailer 2 .

The overall utility function of Retailer 2 is given as follows:

$$
u_{2}= \begin{cases}\pi_{2}-\delta \cdot \max \left\{\pi_{S, 2}-\pi_{2}, 0\right\}-\hat{p}(z) \cdot \rho \cdot\left[\max \left\{\hat{\pi}_{1}(z)-\pi_{2}, 0\right\}\right], & \text { if Accept } \\ 0 & \text { if Reject }\end{cases}
$$


where $\delta \geq 0$ is the distributional fairness parameter and $\rho \geq 0$ is the peer-induced fairness parameter. In equation (2.9), the second term captures Retailer 2's aversion to receiving a smaller profit than the supplier, and the third term captures Retailer 2's aversion to receiving a smaller profit than Retailer 1 . We stress that peer-induced fairness concerns are only relevant between peers, i.e., agents in similar situations. Therefore, the third term in (2.9), which arises when Retailer 2 accepts his offer, makes comparisons against Retailer 1 only when the latter accepts his offer. (Indeed, an offer refused by Retailer 1 may be deemed too high to be a comparable benchmark.) In this spirit, Retailer 2's reference point $\hat{\pi}_{1}(z)$ is the conditional expectation of Retailer 1's profit contingent upon Retailer 1 accepting his offer, and the comparison between $\hat{\pi}_{1}(z)$ and $\pi_{2}$ is weighted by the probability $\hat{p}(z)$ that Retailer 1 has accepted the offer and is indeed a peer.

The utility function (2.9) shows that Retailer 2 has two separate reference points for comparison: the supplier's profit from interacting with himself, i.e., $\pi_{S, 2}$, and Retailer 1's profit conditional on having accepted the offer, i.e., $\hat{\pi}_{1}(z)$. Falling behind each reference point leads to separate disutility terms in (2.9) triggered by different types of fairness concerns. ${ }^{3}$ In this way, our model clearly distinguishes between distributional fairness and peer-induced fairness.

Based on the calculations above, Retailer 2 chooses the best response to maximize his utility as specified in (2.9). Let $p_{2}^{* *}$ denote the optimal retail price that maximizes the first line in (2.9). Then, Retailer 2's best response is to set the retail price as $p_{2}^{* *}$ if the result leads to a positive utility and to reject the supplier's offer otherwise. Note that Retailer 2's best response is influenced by both peer-induced and distributional fairness concerns. In contrast, in the previous section, Retailer 1's best response accounts only for distributional fairness concerns. ${ }^{4}$ The next lemma compares the optimal retail prices of

\footnotetext{
${ }^{3} \mathrm{~A}$ more general model of inequity aversion includes both aversion to disadvantageous inequality (considered in our model) as well as aversion to advantageous inequality (e.g., Fehr and Schmidt, 1999, and Charness and Rabin, 2002). The latter has been found to be empirically absent in a related model (see Ho and $\mathrm{Su}, 2009$ ). Therefore, we omit advantageous inequality in our model.

${ }^{4}$ We do not incorporate peer-induced fairness into Retailer 1's utility because prior research (Ho and $\mathrm{Su} 2009$ ) shows that Retailer 1 does not look ahead and form rational expectation over Retailer 2's expected profit. As a consequence, Retailer 1 does not exhibit peer-induced fairness.
} 
Retailers 1 and 2, conditional on contract acceptance, in response to the same wholesale price offer from the supplier.

Lemma 1. Suppose the supplier offers the same wholesale price $w$ to both retailers, and suppose both retailers accept the offer. Then, the optimal retail prices that maximize the utilities of Retailers 1 and 2 satisfy $p_{1}^{* *}(w) \geq p_{2}^{* *}(w)$.

Proof: See Appendix.

The above lemma states that when peer-induced fairness is in effect $(\rho>0)$, Retailer 1 's price is weakly higher than Retailer 2's price, condition on the same wholesale price offer from the supplier. This is so because Retailer 2 must balance the opposing forces of not being behind the supplier and not being behind Retailer 1 . The first force pushes Retailer 2's price higher while second force pulls it lower. As a consequence, Retailer 2 prices less aggressively than Retailer 1.

Given the systematic differences in the best response functions between Retailers 1 and 2, the supplier can strategically make different wholesale price offers to the retailers in order to optimize her total profit from the retailers. The supplier's problem is forumulated as follows. Recall that the supplier's profit from Retailer 2 depends on the signal $z=w_{1}+\epsilon$, and can be written as

$$
\pi_{S, 2}\left(w_{2}, z\right)= \begin{cases}\left(w_{2}-c\right)\left(a-p_{2}\right), & \text { if Accept } \\ 0 & \text { if Reject }\end{cases}
$$

On the other hand, the supplier's profit from Retailer 1 remains the same as that in the model with only distributional fairness concerns and is given by

$$
\pi_{S, 1}\left(w_{1}\right)= \begin{cases}\left(w_{1}-c\right)\left(a-p_{1}\right), & \text { if Accept } \\ 0 & \text { if Reject }\end{cases}
$$

Therefore, the supplier's objective of the entire game is to maximize

$$
\pi_{S, 1}\left(w_{1}\right)+\mathbf{E}_{z}\left[\pi_{S, 2}\left(w_{2}, z\right)\right]
$$

Based on this model, we can fully characterize the supplier's optimal pricing decisions, taking into account the differences between best responses of Retailers 1 and 2. The 
details are deferred to the Appendix.

Our equilibrium characterization allows us to compare economic outcomes for the retailers as a consequence of peer-induced fairness. In particular, will the second retailer receive a higher or lower wholesale price, and will he earn higher or lower profits? The following propositions answer these questions.

Proposition 2. Suppose $\rho>0$ is not too large. Then, the supplier's wholesale price offer to Retailer 2 is higher than the wholesale price offer to Retailer 1 ; that is, $w_{2}^{* *} \geq w_{1}^{* *}$.

Proof: See Appendix.

Proposition 3. Suppose $\rho>0$ is not too large. Then, Retailer 2 earns less profit (i.e., $\pi_{2} \leq \pi_{1}$ ) and enjoys a smaller market share of the total channel surplus (i.e., $\left.\frac{\pi_{2}}{\pi_{S, 2}+\pi_{2}} \leq \frac{\pi_{1}}{\pi_{S, 1}+\pi_{1}}\right)$ than Retailer 1 .

Proof: See Appendix.

The above results highlight systematic differences between the economic outcomes for Retailers 1 and 2, even though they are identical a priori. Proposition 2 shows that Retailer 2 tends to receive less favorable wholesale price offers, and similarly, Proposition 3 predicts that Retailer 2 will earn lower profits and receive a smaller share of the total channel surplus. In other words, Retailer 2 is in a worse position compared to Retailer 1 , as long as the peer-induced fairness parameter $\rho$ is not too large. ${ }^{5}$ We shall further investigate the comparisons between Retailers 1 and 2 in the empirical analysis below.

\subsection{Testable Hypotheses}

Our analysis above yields Propositions 1-3, which motivate the following testable hypotheses:

\footnotetext{
${ }^{5}$ It is always possible to find an arbitrarily large $\rho$ such that Retailer 2 will reject a wholesale price offer $w_{2}$ whenever the signal realization $z$ satisfies $\hat{\pi}_{1}(z)>\pi_{2}$. In this case, to induce Retailer 2 to accept the offer, the supplier must make a better offer to Retailer 2 than Retailer 1.
} 


\section{Hypothesis 1}

Distributional Fairness Hypothesis: Suppose the supplier makes wholesale price offers to the retailers simultaneously. If retailers have only distributional fairness concerns (i.e., $\delta>0$ and $\rho=0$ ), the wholesales price offer $w_{i}^{*}$ is smaller than wholesale price without fairness concerns, $w_{i}^{o}$.

\section{Hypothesis 2}

Peer-induced Fairness Hypothesis: Suppose the supplier makes wholesale price offers to the retailers sequentially. If Retailer 2 has both distributional and peerinduced fairness concerns (i.e., $\delta>0$ and $\rho>0$ ), then his wholesale price offer, $w_{2}^{* *}$, is higher than the wholesale price of Retailer $1, w_{1}^{* *}$, who has only distributional fairness.

\section{Hypothesis 3}

Order-Dependence Hypothesis: Suppose the supplier makes wholesale price offers to the retailers sequentially. If Retailer 2 has both distributional and peer-induced fairness concerns (i.e., $\delta>0$ and $\rho>0$ ), then he receives a lower profit and enjoys a lower share of total channel surplus than Retailer 1.

\section{Experimental Design}

Our experimental design consists of 2 treatment conditions: 1) Simultaneous and 2) Sequential. In both treatment conditions, we have one supplier selling an identical product through two retailers, each serving his own independent market. The main difference between the treatment conditions is in the manner wholesale price offers are made to the retailers. In the Simultaneous treatment condition, the supplier makes the wholesale prices offers to the retailers simultaneously. In the Sequential treatment condition, she makes these offers sequentially and the second retailer receives a noisy signal of the first wholesale price offer to the first retailer before making his decision. Note that only the second retailer in the Sequential treatment condition is induced to exhibit peer-induced fairness in this experimental design. ${ }^{6}$

\footnotetext{
${ }^{6}$ We could have chosen to test the Distributional Fairness Hypothesis by having a simpler 1-supplier and 1-retailer supply chain instead of the simultaneous treatment condition. We choose a 1 supplier
} 
In both treatment conditions, we set the market size $a=100$ and marginal cost $c=20$. As a result, the optimal wholesale price $w_{i}^{o}=60$ and retail price $p_{i}^{o}=80$. The standard model also predicts that the supplier will make a profit of $\pi_{S, i}^{o}=800$ in each retail market and each retailer will make a profit of $\pi_{i}^{0}=400$ when there is no fairness concern. The noise term $\epsilon$ is uniformly distributed over the following set of discrete values $\{-25,-20,-15,-10,-5,0,5,10,15,20,25\} .{ }^{7}$ We use a standard experimental economics methodology in running our experiments. Specifically, subjects' cash payments are proportional to the profits they make in the experimental task and no deception whatsoever is used in conducting the experiments.

We recruited 135 subjects from a major university in Asia. Sixty six subjects participated in 3 sessions of the Simultaneous treatment and 69 subjects in 4 sessions of the Sequential treatment. The number of subjects in each session is between 15 and 24, and no subjects participated in more than one session. Upon arriving at the laboratory, subjects were randomly seated in cubicles with partitions and were not allowed to talk to each other before and during the experiment. An experimenter read aloud the experimental instructions and subjects were given a chance to clarify questions in private. In addition, an understanding check quiz was conducted to ensure that all subjects truly understood the instructions. Every subject who showed up passed the understanding check and participated in the experiments. See Appendix for the experimental instruction used in the Sequential treatment condition.

Each experiment consisted of 12 identical decision rounds. In each round, subjects were randomly re-grouped into triplets and randomly assigned roles of either supplier, retailer 1, or retailer 2. Anonymity and random-matching protocol were used in order to minimize any reciprocal or reputation building behaviors. In each round, the supplier makes and 2-retailer simultaneous treatment because we want to make the two treatment conditions as similar as possible (e.g. same supply chain structure, the supplier makes the same number of decisions, and members of supply chain make similar level of profits across the treatment conditions, and etc.)

${ }^{7}$ Under this uniform noise structure, it is possible that the signal, $z$, can be negative if actual price offer $w_{1}<25$. In our experiment, this did not happen. That is, all wholesale price offers to Retailer 1 were above 25 . 
wholesale price offers. Retailers either accept or rejected these wholesale price offers and conditional on acceptance they must determine their retail prices. In the sequential treatment condition, Retailer 2 obtains a noisy signal of Retailer 1's wholesale price offer. The experiments were conducted via an online website and subjects' decisions and feedback were all done electronically. We also provided subjects with an excel spreadsheet to allow them to conduct what-if analysis of choosing a price (either wholesale or retail) on their profits (see Lim and Ho, 2008 for a similar experimental design).

The experimental protocol of the Simultaneous treatment condition is as follows:

1. The supplier chooses wholesale price offers for both retailers simultaneously $\left(w_{1}, w_{2}\right)$. Each retailer receives his respective wholesale price offer without receiving a signal of what the other retailer's offer is.

2. Each retailer must independently choose whether or not to accept his respective wholesale price offer from the supplier. Upon acceptance, retailers must choose their respective retail price that will in turn determine the units sold according to the demand function: $d_{i}=100-p_{i}$. If a retailer rejects an offer, both the supplier and the retailer receive zero profit for that specific market.

3. At the end of each round, subjects are informed of their individual decision outcomes and their respective point earnings.

The experimental protocol of the Sequential treatment condition is as follows:

1. The supplier first chooses a wholesale price offer $w_{1}$ to Retailer 1.

2. After receiving the offer, Retailer 1 must first decide whether or not to accept the offer, and upon acceptance he must choose a retail price $p_{1}$. These choices are only revealed to both players at the end of the decision round (i.e., at step 6 of the experimental protocol). ${ }^{8}$

\footnotetext{
${ }^{8}$ We do not immediately reveal Retailer 1 's decisions to the supplier. This helps to avoid learning within a round by the supplier, and also to guard against potential wealth effects (e.g., the supplier becomes more/less generous to Retailer 2 after learning that a good/bad deal has been made with Retailer 1).
} 
3. A signal is generated by adding a random number to the first wholesale price offer $w_{1}$. The value of the random number is drawn from the support $\{-25,-20,-15,-10$, $-5,0,5,10,15,20,25\}$ with each value equally likely to occur. The signal is made known to both the supplier and Retailer $2 .^{9}$

4. The supplier chooses a wholesale price offer $w_{2}$ to retailer 2 .

5. Retailer 2 must now decide whether or not to accept the offer. Upon acceptance, Retailer 2 must choose a retail price $p_{2}$.

6. At the end of each round, subjects are informed of their individual decision outcomes and their respective point earnings.

Each experiment lasted for about one and half hours. Monetary payment was the only incentive used in the experiment: Subjects were paid a $\$ \$ 5$ show-up fee for arriving on-time, and $\mathrm{S} \$ 1.6$ per 1,000 points in profits they earned in the experiment. Subjects received on average $\mathrm{S} \$ 19.2$ with minimum payment of $\mathrm{S} \$ 14.1$ and maximum of $\mathrm{S} \$ 22.8 .^{10}$

\section{Hypothesis Testing and Estimation Results}

\subsection{Summary Statistics}

Table 1 reports the summary statistics of subjects' decisions and profit outcomes.

The left panel of Table 1 shows data from the Simultaneous treatment condition and the right panel shows data from the Sequential treatment condition. In the Simultaneous treatment condition, the average wholesale price offers were 57.80 and 57.67 for Retailers 1 and 2 respectively. The retail prices were similar and the percentages of acceptance were also close. Conditional on acceptance, the average retail prices were 80.09 and 79.70

\footnotetext{
${ }^{9}$ Retailer 2 is also asked to report his guess of what the wholesale price to Retailer 1 is and is rewarded 100 points for a correct guess (see Ho and Su, 2009 for a similar design). Retailer 2 is only told whether her guess is correct or not after the decision round is completed (i.e., at step 6 of the experimental protocol).

${ }^{10}$ In this university, the payment rate for research assistance is $\$ 8.7$ per hour. Hence our payment rate is about $50 \%$ higher than their typical rate of payment.
} 
Table 1: Subjects' Decisions and Profit Outcomes

\begin{tabular}{|c|c|c|c|c|}
\hline & \multicolumn{2}{|c|}{ Simultaneous } & \multicolumn{2}{|c|}{ Sequential } \\
\hline & Retailer1 & Retailer 2 & Retailer 1 & Retailer 2 \\
\hline Decision variables & $(\mathrm{N}=264)$ & $(\mathrm{N}=264)$ & $(\mathrm{N}=276)$ & $(\mathrm{N}=276)$ \\
\hline Wholesale price $\left(w_{i}\right)$ & $57.80(8.03)$ & $57.67(7.73)$ & $57.47(8.38)$ & $58.57(7.88)$ \\
\hline$w_{2}-w_{1}$ & \multicolumn{2}{|c|}{$-0.12(5.06)$} & \multicolumn{2}{|c|}{$1.10(5.93)$} \\
\hline Acceptance $(\%)$ & $95.08(21.68)$ & $93.94(23.91)$ & $96.01(19.60)$ & $95.65(20.43)$ \\
\hline Retail price $\left(p_{i}\right)$ & $80.09(5.19)$ & $79.70(4.87)$ & $80.17(7.09)$ & $80.50(5.97)$ \\
\hline $\begin{array}{l}\text { Performance variables } \\
\text { (upon acceptance) }\end{array}$ & $(\mathrm{N}=251)$ & $(\mathrm{N}=248)$ & $(\mathrm{N}=264)$ & $(\mathrm{N}=263)$ \\
\hline Supplier profit & $711.03(134.07)$ & $723.28(126.54)$ & $698.96(177.22)$ & $710.03(169.85)$ \\
\hline Retailer profit $\left(\pi_{i}\right)$ & $458.63(159.16)$ & $465.08(152.05)$ & $448.41(183.83)$ & $429.40(171.87)$ \\
\hline$\pi_{2}-\pi_{1}$ & \multicolumn{2}{|c|}{$5.09(116.47)$} & \multicolumn{2}{|c|}{$-20.92(149.56)$} \\
\hline Retailer share $\left(m_{i} \%\right)$ & $38.53(8.91)$ & $38.52(8.16)$ & $38.62(10.48)$ & $36.94(9.89)$ \\
\hline$m_{2}-m_{1}$ & \multicolumn{2}{|c|}{$-0.02(6.89)$} & \multicolumn{2}{|c|}{$-1.78(9.55)$} \\
\hline
\end{tabular}

Notes: Standard deviations are reported in parentheses.

respectively. The average retailers' profits were 458.63 and 465.08 and they represented $38.53 \%$ and $38.52 \%$ of the total channel profit respectively. As the table shows, the differences between wholesale price offers, retailer profits, and retailers' shares of channel surplus were close to zero.

Similarly, for the Sequential treatment condition, the right panel of Table 1 has 2 columns, one for each retailer. The average wholesale price offers were 57.47 and 58.57 for Retailers 1 and 2 respectively. While the percentage of acceptance and retail prices were about the same for both retailers, their profits were quite different. Retailer 2 appeared to have made a lower profit than Retailer 1 (448.41 versus 429.40) and enjoyed a lower share of the total channel surplus (38.62\% versus 36.94\%). The differences between Retailers 1 and 2 in the sequential condition are more pronounced than those in the simultaneous treatment. 
Table 2: Tests of Hypotheses

\begin{tabular}{lcc}
\hline Hypothesis & Wilcoxon signed-rank test $(z$-score $)$ & $p$-value \\
\hline $\begin{array}{l}\text { 1. Distributional fairness } \\
\quad w_{i}<60\end{array}$ & $-8.38(\mathrm{~N}=528)$ & 0.0000 \\
2. Peer-induced fairness & & \\
$\quad w_{1}<w_{2}$ & $-3.768(\mathrm{~N}=276)$ & 0.0002 \\
3. Order-dependence & & \\
$\quad$ upon both acceptances $)$ & & \\
(1) $\pi_{2}<\pi_{1}$ & $3.507(\mathrm{~N}=253)$ & 0.0022 \\
(2) $\frac{\pi_{2}}{\pi_{2}+\pi_{S, 2}}<\frac{\pi_{1}}{\pi_{1}+\pi_{S, 1}}$ & $3.897(\mathrm{~N}=253)$ & 0.0001 \\
\hline
\end{tabular}

\subsection{Hypothesis Testing}

Table 2 reports Wilcoxon sign-ranked tests with corresponding $p$-values for our three hypotheses.

1. H1: Distributional Fairness Hypothesis: To test this hypothesis, we use the data from the Simultaneous treatment condition. As expected (see Table 1), there is no difference between the wholesale price offers between the two retailers $(N=264$, Wilcoxon test, $p=0.44)$. Hence, we pool the data from both retailers to test the hypothesis. Wilcoxon signed-rank test suggests that the wholesale price offers are significantly lower than 60 (Wilcoxon test, $p<0.0001$ ). Hence H1 is supported. To control for potential learning effects or trends in subjects' decisions, we used a first-order autoregressive model $\Delta w_{i, t}=\beta_{0}+\beta_{1} \Delta w_{i, t-1}$, where $\Delta w_{i, t}=60-w_{i, t}$ is the difference between the optimal wholesale price without fairness concern and the wholesale price offer in round $t$ and $\Delta w_{i, t-1}$ is the difference in round $t-1$. The estimates are $\hat{\beta}_{0}=2.32$ and $\hat{\beta}_{1}=-0.04$, with clustered standard errors of $0.63(p=0.001)$ and $0.05(p=0.432)$ respectively. The value of $\beta_{0}$ suggests that wholesale price offers remain to be statistically lower than 60 . Since $\beta_{1}$ is not statistically different from 0 , there is minimal learning in the supplier's wholesale price decision over time.

2. H2: Peer-Induced Fairness Hypothesis: To test this hypothesis, we examine whether the difference in wholesale price offers between Retailers 2 and 1 (i.e., $w_{2}-w_{1}$ ) in the sequential treatment condition is higher than 0 . Wilcoxon signed-rank test suggests 
that this is indeed the case $(p=0.0002)$. Hence, the hypothesis is supported. Again, to control for potential learning effects or trends, we used a first-order autoregressive model $\Delta w_{t}=\beta_{0}+\beta_{1} \Delta w_{t-1}$, where $\Delta w_{t}=w_{2, t}-w_{1, t}$ is the difference in the wholesale price offers in round $t$ and $\Delta w_{t-1}$ is the difference in round $t-1$. The estimates are $\hat{\beta}_{0}=1.18$ and $\hat{\beta}_{1}=-0.08$ and clustered standard errors are $0.36(p=0.002)$ and $0.08(p=0.333)$ respectively. The value of $\beta_{0}$ suggests that the difference in wholesale price offers remains statistically higher than 0 . Since $\beta_{1}$ is not statistically different from 0 , there is minimal learning in the supplier's wholesale price decision over time.

3. H3: Order-Dependence Hypothesis: To test this hypothesis, we examine whether the differences in retailer's profits and market shares between Retailers 2 and 1 (i.e., $\pi_{2}-\pi_{1}$ and $\left.\frac{\pi_{2}}{\pi_{2}+\pi_{s, 2}}-\frac{\pi_{1}}{\pi_{1}+\pi_{s, 1}}\right)$ in the sequential treatment condition are lower than 0 . Wilcoxon signed-rank tests suggest that the differences in retailers' profits and shares of the total channel profit are indeed statistically lower than $0(p=0.0022$ and $p=0.0001$ respectively). Hence H3 is supported. To control for learning effects or time trends, we used first-order autoregressive models as follow: ${ }^{11}$

(a) $\Delta \pi_{t}=\beta_{0}+\beta_{1} \Delta \pi_{t-1}$, where $\Delta \pi_{t}=\pi_{2, t}-\pi_{1, t}$ is the difference between retailers' profit in round $t$ and $\Delta \pi_{t-1}$ is the same difference in round $t-1$. The estimates are $\hat{\beta}_{0}=-18.99$ and $\hat{\beta}_{1}=-0.05$ with clustered standard errors $9.09(p=$ $0.038)$ and $0.06(p=0.351)$;

(b) $\Delta m_{t}=\beta_{0}+\beta_{1} \Delta m_{t-1}$, where $\Delta m_{t}=\frac{\pi_{2, t}}{\pi_{2, t}+\pi_{S, 2, t}}-\frac{\pi_{1, t}}{\pi_{1, t}+\pi_{S, 1, t}}$ is the difference between retailers' share of total channel profit in round $t$ and $\Delta m_{t-1}$ is the same difference in round $t-1$. The estimates are $\hat{\beta}_{0}=-2.03$ and $\hat{\beta}_{1}=-0.04$ with clustered standard errors $0.006(p=0.001)$ and $0.04(p=0.290)$.

In both cases, $\beta_{0}$ remain statistically lower than 0 . Since $\beta_{1}$ is statistically not different from 0 , we conclude that there is no significant trend in these performance measures over time.

In summary, the experimental results suggest that all three hypotheses are supported.

\footnotetext{
${ }^{11}$ Since each observation in the two regressions involves decisions by two retailers, we cluster standard errors by the unit that has the same two subjects playing the role of retailers.
} 


\subsection{Structural Estimation}

Our model has two key behavioral parameters, the distributional fairness parameter $\delta$ and the peer-induced fairness parameter $\rho$. In round $t$, we observe the following individual decisions in triplet $j, w_{1 j t}, w_{2 j t}, \mathcal{I}_{1 j t}$ (Retailer 1's acceptance), $\mathcal{I}_{2 j t}$ (Retailer 2's acceptance), and $p_{1 j t}$ and $p_{2 j t}$ conditional on acceptance. Note here $\mathcal{I}_{1 j t}$ and $\mathcal{I}_{2 j t}$ equal to 1 for acceptance and 0 for rejection. We assume normal error terms for wholesale and retail pricing decisions as follows,

$$
\begin{aligned}
& w_{1 j t,}=w_{1}^{* *}+\epsilon_{1}, \\
& w_{2 j t,}=w_{2}^{* *}+\epsilon_{2}, \\
& p_{1 j t,}=p_{1}^{* *}\left(w_{1 j t}\right)+\epsilon_{R 1}, \\
& p_{2 j t,}=p_{2}^{* *}\left(w_{2 j t}\right)+\epsilon_{R 2} .
\end{aligned}
$$

Here $\epsilon_{\ell} \sim \mathcal{N}\left(0, \sigma_{\ell}^{2}\right)$ for $\ell=1,2, R 1, R 2$. The probability density functions for the pricing decisions are donated by $\phi_{1}, \phi_{2}, \varphi_{1}$, and $\varphi_{2}$ respectively. Another parameter of the model, $\sigma$ is the standard deviation of Retailer 2's prior belief of $w_{1}$. Retailers' acceptance decisions follow a Logit choice model with their utility as the independent variable:

$$
\begin{aligned}
& A_{1 j t}=\frac{e^{\left(c_{1}+\beta_{1} \cdot u_{1 j t}^{*}\right)}}{1+e^{\left(c_{1}+\beta_{1} \cdot u_{1 j t}^{*}\right)}}, \\
& A_{2 j t}=\frac{e^{\left(c_{2}+\beta_{2} \cdot u_{2 j t}^{*}\right)}}{1+e^{\left(c_{2}+\beta_{2} \cdot u_{2 j t}^{*}\right)}} .
\end{aligned}
$$

where $c_{i}, \beta_{i}, i=1,2$, are the constants and coefficients of the Logit model, respectively. $u_{1 j t}^{*}\left(u_{2 j t}^{*}\right)$ is the optimal utility if the Retailer 1 (Retailer 2 ) chooses best response assuming acceptance. The joint likelihood function for all decisions can be written as follows:

$$
\begin{array}{r}
\prod_{j} \prod_{t}\left\{\begin{array}{l}
\quad \phi_{1}\left(w_{1 j t}\right) \cdot\left[\mathcal{I}_{1 j t} \cdot A_{1 j t} \cdot \varphi_{1}\left(p_{1 j t}\right)+\left(1-\mathcal{I}_{1 j t}\right) \cdot\left(1-A_{1 j t}\right)\right] \\
\cdot \phi_{2}\left(w_{2 j t}\right) \cdot\left[\mathcal{I}_{2 j t} \cdot A_{2 j t} \cdot \varphi_{2}\left(p_{2 j t}\right)+\left(1-\mathcal{I}_{2 j t}\right) \cdot\left(1-A_{2 j t}\right)\right]
\end{array}\right\}
\end{array}
$$

which is maximized over the whole parameter space of $\delta, \rho, \sigma, \sigma_{1}, \sigma_{2}, \sigma_{R 1}, \sigma_{R 2}, \beta_{1}, \beta_{2}$, $c_{1}$, and $c_{2}$. 
We estimate the full model and two nested models: (1) the basic model without any fairness concerns, that is, $\delta=\rho=0$; (2) the model with distributional fairness only, that is, $\delta>0$ and $\rho=0$. Table 3 shows the estimation results. The two nested models are strongly rejected based on general likelihood principle $\left(\chi^{2}=146.70, p<10^{-31}\right.$ and $\chi^{2}=$ $69.50, p<10^{-15}$, respectively). Thus, both the distributional and peer-induced fairness parameters are important in describing the actual behaviors. The estimated peer-induced fairness parameter $(\rho=6.5380)$ appears more salient than the distributional fairness parameter $(\delta=0.1043)$ in determining Retailer 2 's decisions, because the magnitude of peer comparison between the two retailers is smaller than that of vertical comparison between the retailer and the supplier.

Table 3: Structural Estimation Results

\begin{tabular}{cccc}
\hline Parameters & Model without fairness & Distributional fairness only & Full model \\
\hline$\delta$ & - & 0.0670 & 0.1043 \\
$\rho$ & - & - & 6.5380 \\
\hline$\sigma$ & - & - & 9.2088 \\
$\sigma_{1}$ & 8.7340 & 8.3615 & 8.3663 \\
$\sigma_{2}$ & 7.9907 & 7.9165 & 6.9119 \\
$\sigma_{R 1}$ & 5.3079 & 5.0537 & 5.0256 \\
$\sigma_{R 2}$ & 4.5219 & 4.3322 & 4.3878 \\
$\beta_{1}$ & 0.0075 & 0.0071 & 0.0069 \\
$\beta_{2}$ & 0.0055 & 0.0051 & 0.0009 \\
$c_{1}$ & 0.2089 & 0.5206 & 0.6634 \\
$c_{2}$ & 0.8767 & 1.1461 & 3.2672 \\
\hline $\mathrm{LL}$ & -3632.82 & -3594.22 & -3559.47 \\
\hline
\end{tabular}

\section{$5 \quad$ Interpreting the Estimated Structural Model}

To gain further insight into the estimated structural model, we present some illustrative examples. In these numerical examples, we consider the market scenario used in our laboratory experiments (i.e., market size $a=100$ and marginal cost $c=20$ ) and use the maximum likelihood estimates obtained above (i.e., $\delta=0.1043, \rho=6.5380, \sigma=9.2088$ ). With these parametric values, we can numerically compute the equilibria of the 3 models 
presented in Sections 2.2 to 2.4. Please refer to Table 4 as we discuss these equilibrium results.

Table 4: Differences in Wholesale Price Offers, Retail Prices, Retailers' Profits, and Supplier's Profits from Retailers Conditional on Signal Realization

\begin{tabular}{|c|c|c|c|c|c|c|c|c|c|c|}
\hline \multirow[t]{2}{*}{ Model I } & \multicolumn{10}{|c|}{$\delta=0, \quad \rho=0$} \\
\hline & \multicolumn{3}{|c|}{$w_{i}^{*}=60, \quad p_{i}^{* *}=80$} & \multirow{2}{*}{\multicolumn{3}{|c|}{$\begin{array}{l}\pi_{i}=400, \quad \pi_{S, i}=80 \\
\delta=0.1043, \quad \rho=0\end{array}$}} & \multicolumn{3}{|c|}{$\frac{\pi_{i}}{\pi_{i}+\pi_{S, i}}=33.33 \%$} & \\
\hline \multirow[t]{2}{*}{ Model II } & & & & & & & & & & \\
\hline & & ${ }_{i}^{*}=56.55, \quad p_{i}^{* *}$ & $=80$ & $\pi_{i}=46$ & 9.04 & $\pi_{S, i}=7$ & 30.96 & $\frac{\pi_{i}}{\pi_{i}+\pi_{S, i}}$ & $=39.0$ & \\
\hline \multirow[t]{2}{*}{ Model III } & \multicolumn{10}{|c|}{$\delta=0.1043, \quad \rho=6.5380, \quad \sigma=9.2088$} \\
\hline & \multicolumn{2}{|c|}{$w_{1}^{* *}=57.14}$, & $=80.33$ & \multicolumn{4}{|c|}{$\pi_{1}=456.12, \quad \pi_{S 1}=730.77$} & \multicolumn{3}{|c|}{$\frac{\pi_{1}}{\pi_{1}+\pi_{S, 1}}=38.43 \%$} \\
\hline$\epsilon$ & -25 & $-20 \quad-15$ & -10 & -5 & 0 & 5 & 10 & 15 & 20 & 25 \\
\hline$w_{2}^{* *}$ & 54.19 & 57.85 & 58.62 & 58.94 & 59.07 & 59.25 & 59.46 & 59.46 & 60.05 & 62.78 \\
\hline$w_{2}^{* *}-w_{1}^{* *}$ & -2.96 & -0.74 & 1.48 & 1.80 & 1.93 & 2.11 & 2.32 & 2.32 & 2.91 & 5.64 \\
\hline$p_{2}^{* *}$ & 77.33 & 79.18 & 79.58 & 79.74 & 79.80 & 79.89 & 80.00 & 80.00 & 80.30 & 81.68 \\
\hline$\pi_{2}$ & 524.65 & $475.20 \quad 444.04$ & 427.93 & 421.36 & 418.68 & 415.11 & 410.77 & 410.77 & 398.89 & 346.23 \\
\hline$\frac{\pi_{2}}{\pi_{2}+\pi_{S, 2}} \%$ & 40.36 & $\begin{array}{ll}37.72 & 36.04 \\
\end{array}$ & 35.17 & 34.81 & 34.66 & 34.47 & 34.23 & 34.23 & 33.58 & 30.64 \\
\hline$\pi_{2}-\pi_{1}$ & 68.54 & -12.08 & -28.19 & -34.75 & -37.44 & -41.01 & -45.35 & -45.35 & -57.23 & -109.89 \\
\hline$\pi_{S, 2}$ & 775.13 & $784.48 \quad 787.92$ & 788.87 & 789.09 & 789.15 & 789.21 & 789.23 & 789.23 & 789.05 & 783.64 \\
\hline$\pi_{S, 2}-\pi_{S, 1}$ & 44.36 & 57.15 & 58.11 & 58.32 & 58.38 & 58.44 & 58.46 & 58.46 & 58.28 & 52.88 \\
\hline
\end{tabular}

Our first model (Model I) shows the benchmark case where there are no fairness concerns. This special case is obtained from our full model by restricting the fairness parameters $\delta$ and $\rho$ to 0 . In equilibrium, the retailer earns $\pi_{i}=400$ and the supplier earns $\pi_{S, i}=800$, so the retailer captures one-third of total supply chain profit. The corresponding retail price, wholesale price, and marginal cost are 80, 60, and 20 respectively, so the supplier's margin is twice that of the Retailer.

In the second model (Model II), we allow for distributional fairness concerns by setting $\delta=0.1043$ as estimated above while keeping the peer-induced fairness parameter $\rho$ fixed at 0 . In other words, each retailer is averse to being behind the supplier, but interaction with each retailer remains independent as before. Consequently, both retailers are offered the same terms but these terms are better than that in Model I. As Table 4 shows, the 
supplier's wholesale price offer is $w_{i}^{*}=56.55$, which is lower than the corresponding offer of 60 in Model I. These observations reaffirm the validity of the Distributional Fairness Hypothesis. However, the retail price $p_{i}^{*}=80$ remains unchanged so the total supply chain profit remains unchanged. As a result, the retailer earns a larger share of the total supply chain profit (i.e., 39.09\% compared to 33.3\% in Model I) and the supplier's incurs a profit loss of $8.63 \%$ (i.e., from $\pi_{S, i}=800$ to $\pi_{S, i}=730.96$ ).

We now come to our full model (Model III) which incorporates both distributional and peer-induced fairness concerns. Recall that the estimated fairness parameters are $\delta=0.1043$ and $\rho=6.5380$. In addition, our structural estimation yields $\sigma=9.2088$ : this parameter can be interpreted as the inherent uncertainty in the prior of Retailer 2 on the supplier's wholesale price offer $w_{1}$ to Retailer 1 . As $\sigma$ increases, Retailer 2 has a more diffuse prior on $w_{1}$. With this information structure, the supplier's wholesale price offer $w_{2}$ and Retailer 2's retail price both depend on the signal realization. Table 4 shows the equilibrium behavior for all possible signal realizations. Specifically, as the noise term $\epsilon$ varies from -25 to 25 , the supplier's wholesale price offer $w_{2}^{* *}$ to Retailer 2 ranges from 54.19 to 62.78 , with an expected value of 58.73. In contrast, the supplier's wholesale price offer to Retailer 1 is $w_{1}^{* *}=57.14$ (and it is independent of the signal realization). Compared to Retailer 1, Retailer 2 receives a less attractive offer under most signal realizations. ${ }^{12}$ Note that this observation lends further support to the Peer-Induced Fairness Hypothesis. Finally, Retailer 1 makes a profit of $\pi_{1}=456.12$, which is $38.43 \%$ of the total profit. However, Retailer 2's profits $\pi_{2}$ range from 346.23 to 524.65 (with an expected value of 426.69 ) and his profit share ranges from $30.64 \%$ to $40.36 \%$ (with an expected value of $35.08 \%$ ). These comparisons show that Retailer 1 earns about $6.90 \%$ more profit than Retailer 2 and also receives a larger share of total supply chain profit, confirming the Order-Dependence Hypothesis. As for the supplier, he earns more from Retailer 2 $\left(\pi_{S, 2}\right.$ ranges from 775.13 to 783.64 and equals 786.82 in expectation) than from Retailer

\footnotetext{
${ }^{12}$ When the signal realizations are very small (i.e., $\epsilon=-25$ or -20 in our example), Retailer 2 believes that Retailer 1 received an extremely attractive offer, so the inferred expected profit $\hat{\pi}_{1}(z)$ sets an extremely high reference point. In these cases, the estimated peer-induced fairness parameter $\hat{\rho}=6.5380$ is large enough for the peer-induced fairness disutility term $\hat{p}(z) \cdot \rho \cdot\left[\max \left\{\hat{\pi}_{1}(z)-\pi_{2}, 0\right\}\right]$ in (2.9) to dominate. Hence, contrary to Proposition 2, the supplier must lower the wholesale price offer to induce Retailer 2 to accept, so Retailer 2 receives a better offer than Retailer 1.
} 
$1\left(\pi_{S, 1}=730.77\right)$. The latter figure is almost identical to that in Model II. Therefore, as a result of peer-induced fairness concerns, the supplier's earnings from Retailer 2 has increased by about $7.67 \%$.

Besides being consistent with the qualitative predictions of the three hypotheses, the above results provide a quantitative indication of the effects of fairness on economic outcomes. The Distributional Fairness Hypothesis states that distributional fairness concerns induce the supplier to make a more attractive wholesale price offer to the retailers. Our results support this prediction and our estimates suggest that the supplier incurs a profit loss of about 8.63\%. Next, the Peer-Induced Fairness Hypothesis predicts that a supplier making wholesale offers sequentially and facing a second retailer with peerinduced fairness concerns tends to give a less attractive wholesale price offer to that retailer. Our results agree with this prediction and further suggest that peer-induced fairness enables the supplier to regain about $80.91 \%$ of the profit loss described above (i.e., 800 (no fairness) $\rightarrow 730.96$ (distributional fairness) $\rightarrow 786.82$ (distributional and peer-induced fairness)). Finally, the Order-Dependence Hypothesis states that the second retailer with peer-induced fairness concerns earns less than the first retailer without these concerns. Again, our results support this claim and our structural estimates suggest that the earnings differential between the two retailers is $6.45 \%$ on average and can be more than $20 \%$ for very high values of signal realization (e.g., $\epsilon=25$ ).

Remarkably, non-pecuniary fairness concerns can generate significant economic implications. The underlying intuition can be explained as follows. First, consider a single dyadic supply chain in which distributional fairness concerns arise. In the event that the supplier makes an unfair offer (e.g., one in which the supplier retains a lion's share of total profits), the retailer is tempted to punish the supplier. The most drastic punishment would be to reject the offer, which leads to zero profit for both parties, but this is unlikely to occur in equilibrium. A more plausible equilibrium response is for the retailer to overprice, i.e., choose a price higher than the profit-maximizing response to the supplier's wholesale price. A higher price reduces demand. Therefore, the retailer's action reduces his own slice of the pie but shrinks the supplier's slice by even more (since the supplier's margin is higher, given his unfavorable offer). The result is a more equitable distribution 
of profits as preferred by the retailer. In other words, when faced with an unfair offer, the retailer is willing to hurt himself in order to hurt the supplier even more. Such strategic threats keep the supplier in check. Consequently, in equilibrium, the supplier surrenders a larger portion of the total channel profit to the retailer.

Now, we add a second dyadic supply chain to the picture. The second retailer interacts with the same supplier and thus looks to the first retailer as a peer. With peer-induced fairness concerns, the second retailer is averse to falling behind the first retailer. The urge to keep up with the first retailer makes the second retailer less willing to sacrifice some profit to punish the supplier for an unfavorable offer (as described above). As a result, the supplier can indeed charge the second retailer a higher wholesale price and leave him a smaller fraction of the total supply chain profit. We see that the two types of fairness concerns interact as follows: peer-induced fairness concerns partially neutralize the effect of distributional fairness concerns in attaining equitable profit sharing between the supplier and retailer. Therefore, the two types of fairness concerns have opposite effects: distributional fairness benefits the retailer at the expense of the supplier, but peer-induced fairness benefits the supplier at the expense of the second retailer.

\section{Managerial Implications}

Our research suggests that the supplier benefits when peer-induced fairness concerns are salient to the second retailer. Next, we investigate how the supplier's profits from Retailer 2 vary with the magnitude of the peer-induced fairness parameter $\rho$. Figure 1 plots the supplier's profits as $\rho$ increases, while keeping all other parameters fixed at the estimated values. The dashed curve shows the benchmark case where the retailer is concerned only with distributional fairness but not peer-induced fairness (i.e., Model II) and the solid curve displays the results with both distributional and peer-induced fairness (i.e., Model III), averaged over all signal realizations. Comparing these two curves confirms that peerinduced fairness indeed makes the supplier better off and increasingly so as $\rho$ increases. Intuitively, as $\rho$ increases, Retailer 2 becomes more concerned with keeping up with his peer retailer and is thus less willing to punish the supplier for an unattractive offer. The supplier takes advantage of this aversion-to-behind behavior and ends up making more 
from Retailer 2.

However, this relationship does not always hold in general. We find that the supplier's profits may decrease with $\rho$ at very high levels of uncertainty in Retailer 2's prior, $\sigma$. For example, the dotted curve in Figure 1 shows the what-if results of Model III if the uncertainty in the prior were to be $\sigma=30$ (note that the estimated value of $\sigma$ is 9.2088). In this hypothetical scenario, the supplier's profits steadily decrease as $\rho$ increases after $\rho=1$. As discussed in Section 2.4, the reason is that the supplier must make low wholesale price offers to ensure Retailer 2's acceptance when he has a very strong peer-induced fairness concern. In summary, we conclude that as long as the uncertainty in the prior is not too high, the supplier benefits economically as the peer-induced fairness parameter increases. ${ }^{13}$

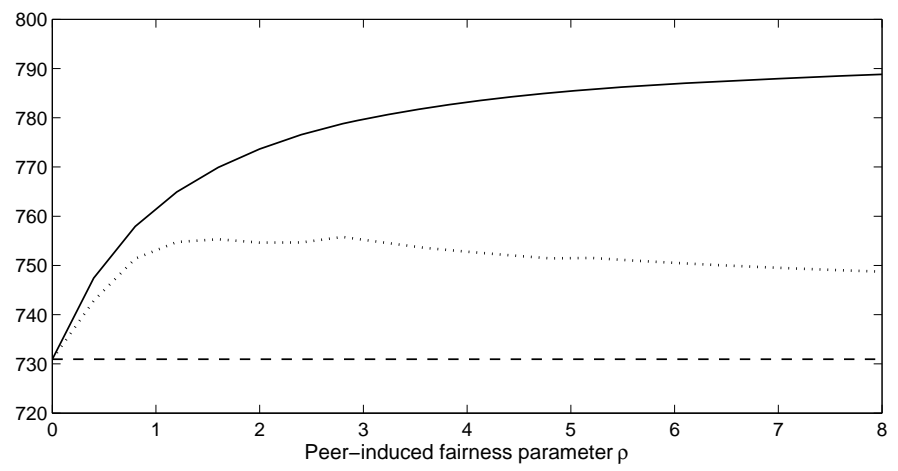

Figure 1: Supplier's profits as $\rho$ varies in Model II (dashed curve), Model III (solid curve) and Model III with $\sigma=30$ (dotted curve).

The above findings suggest that uncertainty in Retailer 2's prior hurts the supplier. This conjecture seems intuitively plausible. Since peer-induced fairness is effective insofar as Retailer 1 serves as a reference point for Retailer 2, the supplier prefers this reference point to be as clear or compelling as possible. Figure 2 plots the supplier's profits as the uncertainty in prior parameter $\sigma$ increases: similar to Figure 1, the dashed curve corre-

\footnotetext{
${ }^{13}$ Note that the suppler's profit curves might not be perfectly smooth. This is so because the retailer utility functions have kinks (due to existence of the two types of fairness concerns) and these kinks can cause supplier's profit function to exhibit discontinuities in its first-order derivative.
} 
sponds to Model II (with only distributional fairness) and the solid curve corresponds to Model III (with both types of fairness). Our results shows that the supplier's gains in profits due to peer-induced fairness decreases with $\sigma$. These results confirm that peerinduced fairness becomes less effective at high levels of uncertainty in Retailer 2's prior belief. Put differently, the supplier is increasingly better off as Retailer 2 becomes more confident in his estimate of Retailer 1's wholesale price offer.

Why does uncertainty in Retailer 2's prior hurt the supplier? When uncertainty in the prior is high, extreme signal realizations are weighted more heavily by Retailer 2. For example, suppose the equilibrium offer to the first retailer is $w_{1}^{* *}=60$ but Retailer 2 observes a highly perturbed signal value of $z=85$. When $\sigma$ is small, Retailer 2's posterior remains close to his prior (i.e., around 60), but when $\sigma$ is large, Retailer 2's posterior shifts more closely toward the signal value of 85 . Such extreme signal realizations are detrimental to the supplier. On one hand, extremely high signals (indicating that Retailer 1 received a very high wholesale price) suggest to Retailer 2 that Retailer 1 most likely made a very low profit or even rejected that offer and thus ceases to be a comparable peer, so peer-induced fairness concerns do not operate. On the other hand, extremely low signals (indicating that Retailer 1 received a very low wholesale price and thus enjoyed a high profit) trigger peer-induced fairness concerns that are so strong as to prompt Retailer 2 to take the drastic course of rejecting the offer in entirety, unless the supplier compensates Retailer 2 with an unusually favorable offer. In both cases, the supplier's profits go down. Thus, high levels of uncertainty in Retailer 2's prior hurt the supplier.

Next, we investigate the impact of the distributional fairness parameter $\delta$. Is peer-induced fairness more effective at high or low values of $\delta$ ? To answer this question, we compute the supplier's equilibrium profits as $\delta$ varies and show our results in Figure 3. As in the previous figures, the dashed curve corresponds to Model II (with only distributional fairness) and the solid curve corresponds to Model III (with both types of fairness). Again, we see that the solid curve lies above the dashed curve, indicating that peer-induced fairness increases supplier profits. However, these profit gains diminish to zero as $\delta$ goes to zero and as $\delta$ grows large (i.e., beyond $\delta=1.0$ in this example). The reasons are as 


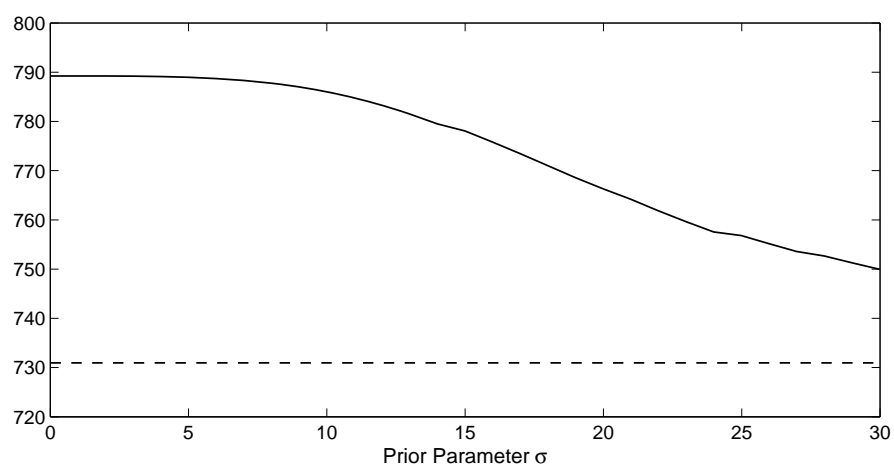

Figure 2: Supplier's profits as $\sigma$ varies in Model II (dashed curve) and Model III (solid curve).

follows. When $\delta$ is close to zero, the equilibrium outcomes in both Models II and III are almost identical to those in Model I (i.e., the supplier's profits are close to 800) because players focus on profit maximization. Since financial incentives overshadow fairness concerns, peer comparisons do not significantly impact outcomes. On the other hand, when $\delta$ is large, distributional fairness concerns are so dominant that any deviation from the 50-50 benchmark cannot be tolerated by either retailer. In Model II, the equilibrium outcome when $\delta$ is large involves a wholesale price of 46.67, a retail price of 73.33 , and an equal profit of 711.11 for both the retailer and the supplier. (Note that this is the equilibrium outcome in Proposition 1 for $\delta \geq 1 / 7$.) In Model III, when $\delta$ is large, the supplier may have to contend with even lower profits to compensate for potential peerinduced fairness concerns, especially under extreme signal realizations. Therefore, we conclude that peer-induced fairness concerns can effectively improve supplier profits only when the distributional fairness parameter is neither too large nor too small.

Finally, we compare our results to a related study by $\mathrm{Ho}$ and $\mathrm{Su}$ (2009). Ho and $\mathrm{Su}$ study peer-induced fairness in a similar setup with two retailers interacting with the same supplier, but each retailer-supplier interaction is modeled as an ultimatum game rather than the pricing game considered here. In an ultimatum game, the total pie size is fixed (i.e., retail price and hence total demand are fixed), so the supplier's wholesale price offer translates into a particular division of the pie, which the retailer can only accept or reject. In this paper, we generalize the ultimatum setup to a pricing game. By 


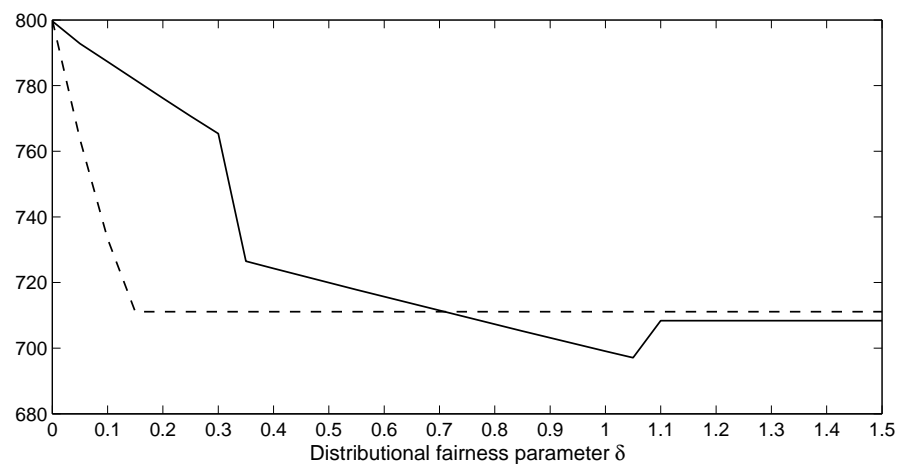

Figure 3: Supplier's profits as $\delta$ varies in Model II (dashed curve) and Model III (solid curve).

doing so, we significantly enrich our model because endowing the retailer with pricing power implies that the total pie size will be endogenously determined in equilibrium. As discussed above, endogenous pie sizes significantly change players' strategies in this game.

The most dramatic difference between these two studies is the reversal of profit comparisons between the retailers. In Ho and $\mathrm{Su}$ (2009), Retailer 2 on average makes more profit than Retailer 1. However, in this paper, the opposite holds. The reason is as follows. With peer-induced fairness concerns, Retailer 2 naturally uses Retailer 1 as a reference point, but this reference point plays a different role in the two studies. In Ho and $\mathrm{Su}$ (2009), the ultimatum game ends once the retailer accepts or rejects the supplier's offer; therefore, the reference point prompts Retailer 2 to accept only if the offer is not too inferior relative to that of Retailer 1 . Put differently, a higher offer to Retailer 1 makes the supplier liable to make a comparatively attractive offer to Retailer 2. Consequently, to avoid creating too high a reference point, the supplier makes a worse offer to Retailer 1 than Retailer 2. In this paper, the game does not end after the retailer accepts the wholesale price contract. Conditional on contract acceptance, the retailer goes on to set the retail price, and it is at this stage of the game where the reference point affects Retailer 2's behavior. Since Retailer 2 has already accepted the contract, the reference point now works against his favor by prompting him to attain similar profits as Retailer 1. This aversion to being behind Retailer 1 restricts Retailer 2's ability to punish the supplier for making bad wholesale price offers. Recognizing this factor, the supplier in- 
deed leaves Retailer 2 with smaller profits than Retailer 1.

The above discussion suggests that one way Retailer 2 can turn his positional disadvantage into an advantage is to make a priori retail price commitments. By doing so, Retailer 2 effectively changes the current game setup into the one studied by $\mathrm{Ho}$ and $\mathrm{Su}$ (2009), where the second retailer is always treated better. In this way, the retailer may secure a better profit by voluntarily giving up his pricing power.

\section{Conclusions}

We examine the interaction of distributional and peer-induced fairness in price contract design in a 1-supplier, 2-retailer supply chain. We show that if the supplier makes wholesale price offers simultaneously, it is optimal for the supplier to make a lower wholesale price offer when the retailers have distributional fairness concerns than when they have no fairness concerns. Surprisingly, if the supplier makes the wholesale price offers sequentially (and the second retailer has both distributional and peer-induced fairness concerns), it is optimal for the supplier to make the second wholesale price offer higher than the first wholesale price offer. As a consequence, the second retailer makes a lower profit and enjoys a lower share of the total channel surplus when compared to the first retailer. To the best of our knowledge, the set of theoretical results relating to sequential price offers are new to the literature.

We conduct standard economic experiments with subjects motivated by substantial monetary incentives to test our model predictions. The experimental data strongly support our model predictions. Specifically, when the supplier makes wholesale price offers simultaneously, the wholesale prices are lower than that predicted by the standard model (where retailers are purely self-interested), confirming that our subjects indeed have distributional fairness concerns. If wholesale price offers are made sequentially, we find that the second wholesale price offer is indeed higher than the first wholesale price offer, suggesting that the second retailer has peer-induced fairness concerns.

Finally, we structurally estimate our general model using the experimental data. We 
show that the two nested models (i.e., a model without fairness concerns and a model with only distributional fairness concerns) are strongly rejected in favor of the general model. The estimated parameters suggest that peer-induced fairness is more salient than distributional fairness in determining second retailer's pricing behavior.

This research can be extended in several directions. First, the model can be extended with the supplier using a more complex wholesale pricing contract (e.g., quantity discount pricing contract). Second, it may be worthwhile to make the demand function a function of both the sale effort and retail price. For example, the total market size a can be made dependent on a retailer's sale effort and one can then investigate how the optimal sale effort changes as a function of peer-induced fairness concerns. Finally, it may be interesting to extend the game to a setting where the supplier must interact with the retailers repeatedly over time (and each retailer receives information on the other's retail price). The model must then be extended to have both retailers exhibiting peer-induced fairness concerns.

\section{References}

Anderson, E., B. Weitz. 1992. The use of pledges to build and sustain commitment in distribution channels. Journal of Marketing Research 29(1) 18-34.

Avci, B., Z. Loutfi, J. Mihm, E. Belavina, S. Keck. 2012. Comparison as incentive: Newsvendor decisions in a social context. Production and Operations Management forthcoming

Bolton, G. E., A. Ockenfels. 2000. ERC: A theory of equity, reciprocity, and competition. American Economic Review 90(1) 166-193.

Charness , G., M. Rabin. 2002. Understanding social preferences with simple tests. Quarterly Journal of Economics 117(3) 817-869

Cui, T. H., J. S. Raju, Z. J. Zhang. 2007. Fairness and channel coordination. Management Science 53(8) 1303 - 1314. 
Fehr, E., K. M. Schmidt. 1999. A theory of fairness, competition and co-operations. Quarterly Journal of Economics 114(3) 817-868.

Ho, T-H, Lim, N. and Cui, T. 2010. Reference-Dependence in Multi-location Newsvendor Models: A Structural Analysis. Management Science, 56 (11) 1891-1910.

Ho, T.-H., X. Su. 2009. Peer-induced fairness in games. American Economic Review 99(5) 2022-2049

Ho, T.-H., J. Zhang. 2007. Designing price contracts for boundedly rational customers: Does the framing of the fixed fee matter? Management Science 54(4) 686-700.

Lim, N., T.-H. Ho. 2008. Designing price contracts for boundedly rational customers: Does the number of blocks matter? Marketing Science 26(3) 321-326.

Loch, C., Y. Wu. 2007. Behavioral operations management. Foundations and Trends in Technology, Information and Operations Management 1(3) 121-232.

Loch, C., Y. Wu. 2008. Social preferences and supply chain performance: An experimental study. Management Science 54(11) 1835-1849.

Kahneman, D., J. L. Knetsch, R. Thaler. 1986. Fairness as a constraint on profit seeking: Entitlements in the markets. American Economic Review 47(2) 263-291.

Kahneman, D., A. Tversky. 1979. Prospect theory: An analysis of decision under risk. Econometrica 76(4) 728-741.

Kalkanci, B., K. -Y. Chen., F. Erhun. 2011. Contract complexity and performance under asymmetric demand information: An experimental evaluation. Management Science $57(4)$ 689-704.

Katok, E., V. Pavlov. 2012. Fairness in supply chain contracts: A laboratory study. Working paper, University of Texas at Dallas.

Katok, E., T. Olsen, V. Pavlov. 2012. Wholesale pricing under mild and privately known concerns for fairness. Production and Operations Management Forthcoming.

Katok, E., D. Wu. 2009. Contracting in supply chains: A laboratory investigation. Management Science 55(12) 1953-1968. 
Pavlov V., E. Katok. 2012. Fairness and coordination failures in supply chain contracts. Working paper, University of Texas at Dallas.

$\mathrm{Su}, \mathrm{X} .2008$. Bounded rationality in newsvendor models. Manufacturing $\mathscr{E} 3$ Service Operations Management 10(4) 566-589.

Wu, Y., C. Loch, G. Ahmad, 2011. Status and relationships in social dilemmas of teams. Journal of Operations Management 29(7-8) 650-662. 


\section{A Instructions}

This is an experiment about economic decision-making. The instructions are simple; and if you follow them carefully and make appropriate decisions, you may earn a considerable amount of money which will be paid to you in cash at the end of the experiment. It is important that you do not look at the decisions of others, and that you do not talk, laugh, or make noises during the experiment. You will be warned if you violate this rule the first time. If you violate this rule twice, you will be asked to leave the room immediately and your cash earnings will be $\$ 0$.

The experiment will consist of 12 decision rounds. In each round, participants will be randomly assigned into groups of triplets. One player of each triplet will be assigned the role of SUPPLIER, and the remaining two players in the triplet will be assigned the role of RETAILERS (RETAILER 1 and RETAILER 2, respectively). You have an equal chance of playing the role of SUPPLIER, RETAILER 1 or RETAILER 2 in each round. Your role will vary from round to round.

The SUPPLIER sells an identical product through RETAILER 1 and RETAILER 2 to two separate groups of customers. The SUPPLIER must sequentially decide on a wholesale price offer for each retailer. RETAILERS must decide whether or not to accept their respective price offer, and upon acceptance, choose a retail price at which he/she will sell the product to his/her own customers. The detailed experimental procedure is described below.

\section{Experimental Procedure}

Each decision round consists of five decision stages:

Stage 1: The SUPPLIER chooses a wholesale price offer for RETAILER 1

The SUPPLIER chooses a WHOLESALE PRICE 1 at which to sell to RETAILER 1. The WHOLESALE PRICE 1 must be a positive integer. Since each unit of product costs the SUPPLIER 20 points, the SUPPLIER will earn (WHOLESALE PRICE 1 - 20)x( QUANTITY 1), where the number of unit sold by the SUPPLIER (QUANTITY 1) depends on RETAILER 1's decisions, which is described next.

Stage 2: RETAILER 1 chooses whether or not to accept the SUPPLIER's price offer, and upon acceptance, must choose a retail price

Upon receiving the wholesale price offer from the SUPPLIER, RETAILER 1 must first decide whether or not to accept the wholesale price offer. If RETAILER 1 rejects the offer, both the SUPPLIER and RETAILER 1 earn zero point. Specifically, RETAILER 1 earns zero point for this decision round and the SUPPLIER earns zero point from RETAILER 1 in this decision round (The SUPPLIER may still earn point from RETAILER 2 in this decision round if the latter accepts the formers price offer). 
If RETAILER 1 accepts the price offer, he/she must then choose retail PRICE 1 at which to sell to his/her customers. The retail PRICE 1 must be a positive integer. The number of units sold (QUANTITY 1) is determined by PRICE 1 in the following way:

$$
\text { QUANTITY } 1=100-\text { PRICE } 1 .
$$

For example, if PRICE 1 is 70 , QUANTITY 1 is 30 . If PRICE 1 is 30 , QUANTITY 1 is 70 . Note that these numbers are chosen arbitrarily for illustrative purposes only.

The decision outcomes of Stage 2 (e.g., whether RETAILER 1 accepts or rejects, and retail PRICE 1) will only be revealed to the SUPPLIER at the end of the decision round (after Stage 5 is completed).

Stage 3: RETAILER 2 receives a signal about the WHOLESALE PRICE 1

In this stage, RETAILER 2 will receive a signal of the SUPPLIER's WHOLESALE PRICE 1 offer for RETAILER 1 in stage 1. The signal is generated as follows:

a. A number $\mathrm{X}$ is first randomly drawn from a set of 11 numbers: $-25,-20,-15,-10,-5,0,5,10,15$, 20,25 ; each of which has an equal chance (1/11 chance) of being chosen.

b. The SIGNAL is the sum of $\mathrm{X}$ and actual WHOLESALE PRICE 1, that is,

$$
\text { SIGNAL }=\text { WHOLESALE PRICE } 1+\mathrm{X} .
$$

c. The value of the SIGNAL is announced to both the SUPPLIER and RETAILER 2.

d. Note that we conduct a fresh draw of $\mathrm{X}$ for each triplet in each round.

The following tables provide two concrete examples. The left table shows the case where the SIGNAL value is 50. In this case, there are 11 possible WHOLESALE PRICE 1 offers: it can range from 25 to 75 depending on the value of the random number $\mathrm{X}$. For example, if $\mathrm{X}$ is 0 , then the WHOLESALE PRICE 1 is 50 (since $50=50+0$ ). Similarly, if $X=10$, then the WHOLESALE PRICE 1 is 40 (since $50=40+$ 10). The right table shows the case where the SIGNAL is 70 . Again, there are 11 possible WHOLESALE PRICE 1 offers: in this case, it can range from 45 to 95 depending on the value of the random number of $\mathrm{X}$. Note that the values of the signal in these two examples are chosen only for illustrative purposes. 


\begin{tabular}{ccc}
\hline WHOLESALE PRICE 1 & $\mathrm{X}$ & SIGNAL \\
\hline 75 & -25 & 50 \\
\hline 70 & -20 & 50 \\
\hline 65 & -15 & 50 \\
\hline 60 & -10 & 50 \\
\hline 55 & -5 & 50 \\
\hline 50 & 0 & 50 \\
\hline 45 & 5 & 50 \\
\hline 40 & 10 & 50 \\
\hline 35 & 15 & 50 \\
\hline 30 & 20 & 50 \\
\hline 25 & 25 & 50 \\
\hline
\end{tabular}

\begin{tabular}{ccc}
\hline WHOLESALE PRICE 1 & $\mathrm{X}$ & SIGNAL \\
\hline 95 & -25 & 70 \\
\hline 90 & -20 & 70 \\
\hline 85 & -15 & 70 \\
\hline 80 & -10 & 70 \\
\hline 75 & -5 & 70 \\
\hline 70 & 0 & 70 \\
\hline 65 & 5 & 70 \\
\hline 60 & 10 & 70 \\
\hline 55 & 15 & 70 \\
\hline 50 & 20 & 70 \\
\hline 45 & 25 & 70 \\
\hline
\end{tabular}

After receiving the SIGNAL, RETAILER 2 will be asked to guess what WHOLESALE PRICE 1 is. If RETAILER 2's guess is correct, he or she will receive a total of 100 points as a reward. If RETAILER 2 's guess is wrong, he or she will receive nothing.

Note that whether the RETAILER 2's guess is correct will be revealed to Retailer 2 at the end of the decision round (after Stage 5 is completed).

Stage 4: The SUPPLIER chooses a wholesale price offer for RETAILER 2.

The SUPPLIER will be informed of the value of the same SIGNAL RETAILER 2 receives and the guess RETAILER 2 makes. The SUPPLIER then chooses a WHOLESALE PRICE 2 at which to sell to RETAILER 2. The WHOLESALE PRICE 2 must be a positive integer. Since each unit costs the SUPPLIER 20 points, the SUPPLIER will earn (WHOLESALE PRICE 2 - 20)x(QUANTITY 2), where the number of unit sold by the SUPPLIER (QUANTITY 2) depends on RETAILER 2's decision, which is described next.

Stage 5: RETAILER 2 chooses whether or not to accept the SUPPLIER's price offer, and upon acceptance, must choose a retail price

Upon receiving the wholesale price offer from the SUPPLIER, RETAILER 2 must first decide whether or not to accept the price offer. If RETAILER 2 rejects the offer, both the SUPPLIER and RETAILER 2 earn zero point. Specifically, RETAILER 2 earns zero point for this decision round and the SUPPLIER earns zero point from RETAILER 2 in this decision round (The SUPPLIER may still earn points from RETAILER 1 in this decision round if the latter accepts the former's price offer).

If RETAILER 2 accepts the price offer, he/she must then choose retail PRICE 2 at which to sell to his/her customers. The retail PRICE 2 must be a positive integer. The number of units sold (QUANTITY 2) 
is determined by PRICE 2 in the following way:

$$
\text { QUANTITY } 2=100-\text { PRICE } 2 \text {. }
$$

For example, if PRICE 2 is 70, QUANTITY 2 is 30. If PRICE 2 is 30 , QUANTITY 2 is 70 . Note that these numbers are chosen arbitrarily for illustrative purposes only.

\section{Point Earnings}

The point earning for each player will be calculated as follows:

1. If RETAILER 1 accepts the SUPPLIER's offer, his/her point earning equals to

$$
\text { (PRICE } 1 \text { - WHOLESALE PRICE 1) x (QUANTITY 1). }
$$

That is, the difference between the price RETAILER 1 charges customers and the price at which he/she buys the product from the SUPPLIER multiplied by the quantity sold. Note here QUANTITY $1=100-$ PRICE 1 .

If RETAILER 1 chooses not to accept the offer, then QUANTITY 1 is 0 , and his/her earning is 0 .

2. If RETAILER 2 accepts the SUPPLIER's offer, his/her point earning equals to

(PRICE 2 - WHOLESALE PRICE 2) x (QUANTITY 2).

That is, the difference between the price RETAILER 2 charges customers and the price at which he/she buys the product from the SUPPLIER multiplied by the quantity sold. Note here QUANTITY $2=100-$ PRICE 2 .

If RETAILER 2 chooses not to accept the offer, then QUANTITY 2 is 0 , and his/her earning is 0 .

3. The SUPPLIER earns points from both RETAILER 1 and 2.

The SUPPLIER's point earning from RETAILER 1 equals to

(WHOLESALE PRICE 1 - 20)x(QUANTITY 1).

That is, the difference between the price at which the product is sold to RETAILER 1 and the unit cost of the product multiplied by the quantity sold. If RETAILER 1 does not accept the offer, then QUANTITY 1 is 0 and the earning from RETAILER 1 is 0.

The SUPPLIER's point earning from RETAILER 2 equals to 


\section{(WHOLESALE PRICE 2 - 20) x (QUANTITY 2).}

That is, the difference between the price at which the product is sold to RETAILER 2 and the unit cost of the product multiplied by the quantity sold. If RETAILER 2 does not accept the offer, then QUANTITY 2 is 0 and the earning from RETAILER 2 is 0.

The SUPPLIER's total point earning is the sum of the point earnings from RETAILER 1 and 2, that is,

\section{(WHOLESALE PRICE 1 - 20)x(QUANTITY 1) + (WHOLESALE PRICE 2 - 20)x(QUANTITY 2).}

At the end of each round, the SUPPLIER and both RETAILERS will be informed of their individual decision outcomes and their earnings. The above decision task is repeated for 12 rounds. In each round, each player will be randomly grouped into a triplet and have an equal chance of playing SUPPLIER, RETAILER 1 or RETAILER 2. Also, a fresh draw of random number $\mathrm{X}$ is drawn for each triplet.

\section{Your Dollar Payoffs}

At the end of the experiment, we will sum your point earning in each round to obtain your total point earning. We will then multiply your total point earning by $\$ 0.0016$ to obtain your dollar earning. In addition, we will add to this dollar earning $\$ 5$ show-up fee to determine your final dollar earning. The amount will be paid to you in cash before you leave the experiment today. 


\section{B Appendix}

\subsection{Retailer 1's Best Response Function}

When Retailer 1 experiences distributional fairness, that is, $\pi_{S, 1}-\pi_{1} \geq 0$, or equivalently $p_{1} \leq 2 w_{1}-c$, Retailer 1's utility-maximization problem upon acceptance is given by

$$
\begin{array}{ll}
\max _{p_{1}} & \pi_{1}-\delta \cdot\left(\pi_{S, 1}-\pi_{1}\right) \\
\text { s.t. } & p_{1} \leq 2 w_{1}-c .
\end{array}
$$

The optimal retail price is given by

$$
p_{1}= \begin{cases}\frac{a+w_{1}}{2}+\frac{\delta\left(w_{1}-c\right)}{2(1+\delta)}, & \text { if } w_{1} \geq \frac{a(1+\delta)+c(2+\delta)}{3+2 \delta} \\ 2 w_{1}-c, & \text { otherwise. }\end{cases}
$$

When Retailer 1 does not experience distributional fairness, that is, $\pi_{S, 1}-\pi_{1}>0$, or $p_{1}>2 w_{1}-c$, Retailer 2's utility-maximization problem upon acceptance is given by

$$
\begin{array}{ll}
\max _{p_{1}} & \pi_{1} \\
\text { s.t. } & p_{1}>2 w_{1}-c .
\end{array}
$$

The optimal retail price is given by

$$
p_{1}= \begin{cases}\frac{a+w_{1}}{2}, & \text { if } w_{A}<\frac{a+2 c}{3} \\ 2 w_{1}-c, & \text { otherwise }\end{cases}
$$

Since $\frac{a(1+\delta)+c(2+\delta)}{3+2 \delta}>\frac{a+2 c}{3}$ always holds for $\delta>0$, Retailer 1's optimal retail price can be found by combining the above two cases as follows

$$
p_{1}^{* *}= \begin{cases}\frac{a+w_{1}}{2}+\frac{\delta\left(w_{1}-c\right)}{2(1+\delta)}, & \text { if } w_{1} \geq \frac{a(1+\delta)+c(2+\delta)}{3+2 \delta} \\ 2 w_{1}-c, & \frac{a+2 c}{3} \leq w_{1}<\frac{a(1+\delta)+c(2+\delta)}{3+2 \delta} \\ \frac{a+w_{1}}{2}, & \text { if } w_{1}<\frac{a+2 c}{3}\end{cases}
$$

As a result, Retailer 1's best response is to choose $p_{1}^{* *}$ if this leads to a positive utility and to reject otherwise.

Lemma 2. Retailer 1's profit $\pi_{1}$ and utility $u_{1}$ under the optimal response given in (B.1) decreases in $w_{1}$.

Proof. The properties can be verified by substituting (B.1) into $\pi_{1}$ and $u_{1}$ and take the first-order derivatives with respect to $w_{1}$.

\subsection{Retailer 2's Best Response and Supplier's Pricing Decision}

\subsubsection{Retailer 2's Decision When $w_{2}>a-2 \sqrt{\hat{\pi}_{1}(z)}$}

The difference between Retailer 2's and Retailer 1's problems is that Retailer 2 may experience peerinduced fairness depending on $w_{2}$ and $p_{2}$. Retailer 2 has peer-induced fairness when $\left(a-p_{2}\right)\left(p_{2}-w_{2}\right)<$ 
$\hat{\pi}_{1}(z)$. This inequality always holds when $w_{2}>a-2 \sqrt{\hat{\pi}_{1}(z)}$. We first analyze Retailer 2's best response under this condition, the problem is similar to Retailer 1's problem shown as follows.

When Retailer 2 experiences distributional fairness, that is $\pi_{S, 2}-\pi_{2} \geq 0$, or equivalently, $p_{2} \leq 2 w_{2}-c$, Retailer 2's problem is given by

$$
\begin{array}{ll}
\max _{p_{2}} & \pi_{2}-\delta \cdot\left(\pi_{S, 2}-\pi_{2}\right)-\hat{p}(z) \cdot \rho \cdot\left(\hat{\pi}_{1}(z)-\pi_{2}\right) \\
\text { s.t. } & p_{2} \leq 2 w_{2}-c .
\end{array}
$$

The optimal solution for the unconstrained problem (B.2) is $p_{2}=\frac{a+w_{2}}{2}+\frac{\delta\left(w_{2}-c\right)}{2(1+\delta+\hat{p}(z) \cdot \rho)}$. Constraint (B.3) is satisfied when $w_{2} \geq w_{2}^{I}:=\frac{a(1+\delta+\hat{p} \cdot \rho)+c(2(1+\hat{p} \cdot \rho)+\delta)}{3(1+\hat{p} \cdot \rho)+2 \delta}$. Then Retailer 2's optimal retail price is given by

$$
p_{2}= \begin{cases}\frac{a+w_{2}}{2}+\frac{\delta\left(w_{2}-c\right)}{2(1+\delta+\hat{p}(z) \cdot \rho)}, & \text { if } w_{2} \geq w_{2}^{I} \\ 2 w_{2}-c, & \text { otherwise }\end{cases}
$$

When Retailer 2 does not experience distributional fairness, that is $\pi_{S, 2}-\pi_{2}<0$, or equivalently, $p_{2}>2 w_{2}-c$, Retailer 2's problem is given by

$$
\begin{array}{ll}
\max _{p_{2}} & \pi_{2}-\hat{p}(z) \cdot \rho \cdot\left(\hat{\pi}_{1}(z)-\pi_{2}\right) \\
\text { s.t. } & p_{2}>2 w_{2}-c .
\end{array}
$$

The optimal solution for the unconstrained problem (B.5) is $p_{2}=\frac{a+w_{2}}{2}$. Constraint (B.6) is satisfied when $w_{2}<\frac{a+2 c}{3}$. Then Retailer 2's optimal retail price is given by

$$
p_{2}= \begin{cases}2 w_{2}-c, & \text { if } w_{2} \geq \frac{a+2 c}{3} \\ \frac{a+w_{2}}{2}, & \text { otherwise }\end{cases}
$$

Since $\frac{a(1+\delta+\hat{p}(z) \cdot \rho)+c(2(1+\hat{p}(z) \cdot \rho)+\delta)}{3(1+\hat{p}(z) \cdot \rho)+2 \delta}>\frac{a+2 c}{3}$ always holds for $\delta>0$, Retailer 2's optimal retail price when $w_{2}>a-2 \sqrt{\hat{\pi}_{1}(z)}$ can be found by combining (B.4) and (B.7) as follows

$$
p_{2}^{* *}= \begin{cases}\frac{a+w_{2}}{2}+\frac{\delta\left(w_{2}-c\right)}{2(1+\delta+\hat{p}(z) \cdot \rho)}, & \text { if } w_{2} \geq w_{2}^{I} \\ 2 w_{2}-c, & \frac{a+2 c}{3} \leq w_{2}<w_{2}^{I} \\ \frac{a+w_{2}}{2}, & \text { if } w_{2}<\frac{a+2 c}{3}\end{cases}
$$

As a result, Retailer 2's best response is to choose $p_{2}^{* *}$ if this leads to a positive utility and to reject otherwise.

\subsubsection{Supplier's Decision When $w_{2}>a-2 \sqrt{\hat{\pi}_{1}(z)}$}

Given the best response function (B.8) and conditional on contract acceptance, the optimal wholesale price offer can be found as follows: (1) When $w_{2} \geq w_{2}^{I}$, the supplier's profit function is $\pi_{S, 2}=$ $\left(a-\frac{a+w_{2}}{2}-\frac{\delta\left(w_{2}-c\right)}{2(1+\delta+\hat{p}(z) \cdot \rho)}\right) \cdot\left(w_{2}-c\right)$, and the optimal solution is $w_{2}=\frac{a+c}{2}-\frac{\delta(a-c)}{2(1+2 \delta+\hat{p}(z) \cdot \rho)}$. This solution satisfies the condition $w_{2} \geq w_{2}^{I}$ when $\delta \leq \frac{1+\hat{p}(z) \cdot \rho}{2}$. When $\delta>\frac{1+\hat{p}(z) \cdot \rho}{2}$, the optimal decision in this 
region is $w_{2}=w_{2}^{I}$; (2) When $\frac{a+2 c}{3} \leq w_{2}<w_{2}^{I}$, the supplier's profit is $\pi_{S, 2}=\left(a-2 w_{2}+c\right)\left(w_{2}-c\right)$. The optimal solution is $w_{2}=\frac{a+3 c}{4}$ that is not within the boundaries of $\frac{a+2 c}{3} \leq w_{2}<w_{B}^{I}$, and the optimal wholesale price is $w_{2}=\frac{a+2 c}{3} ;(3)$ When $w_{2}<\frac{a+2 c}{3}$, the supplier's profit is $\pi_{S, 2}=\left(a-\frac{a+w_{2}}{2}\right)\left(w_{2}-c\right)$. The optimal solution is $w_{2}=\frac{a+c}{2}$ that is not within the boundaries of $w_{2} \leq \frac{a+2 c}{3}$, and the optimal wholesale price is $w_{2}=\frac{a+2 c}{3}$.

For (1), the supplier's profit is $\frac{(a-c)^{2}(1+\delta+\hat{p}(z) \cdot \rho)}{8(1+2 \delta+\hat{p}(z) \rho)}$, and for (2) and (3), the supplier's profit is $\frac{(a-c)^{2}}{9}$. Comparing these two potential profits, $\frac{(a-c)^{2}(1+\delta+\hat{p}(z) \cdot \rho)}{8(1+2 \delta+\hat{p}(z) \cdot \rho)}>\frac{(a-c)^{2}}{9}$ when $\delta<\frac{1+\hat{p}(z) \rho}{7}$. Therefore, conditional on contract acceptance, the supplier's optimal decision is

$$
w_{2}^{* *}= \begin{cases}\frac{a+c}{2}-\frac{\delta(a-c)}{2(1+2 \delta+\hat{p}(z) \cdot \rho)}, & \text { if } \delta<\frac{1+\hat{p}(z) \cdot \rho}{7} \\ \frac{a+2 c}{3}, & \text { otherwise }\end{cases}
$$

\subsubsection{Retailer 2's Decision When $w_{2} \leq a-2 \sqrt{\hat{\pi}_{1}(z)}$}

When $w_{2} \leq a-2 \sqrt{\hat{\pi}_{1}(z)}$, we consider four scenarios of Retailer 2's utility maximization problem according to possible fairness concerns experienced by Retailer 2 .

Case 1. Retailer 2 is neither behind Retailer 1 nor behind the supplier, or equivalently, (1) $\left(a-p_{2}\right)$. $\left(p_{2}-w_{2}\right)>\hat{\pi}_{1}(z)$, i.e., $\frac{a+w_{2}-\sqrt{\left(a-w_{2}\right)^{2}-4 \hat{\pi}_{1}(z)}}{2}<p_{2}<\frac{a+w_{2}+\sqrt{\left(a-w_{2}\right)^{2}-4 \hat{\pi}_{1}(z)}}{2} ;(2)\left(a-p_{2}\right) \cdot\left(p_{2}-w_{2}\right)>$ $\left(a-p_{2}\right)\left(w_{2}-c\right)$, i.e., $p_{2}>2 w_{2}-c$. Then Retailer 2 's problem is given by

$$
\begin{array}{ll}
\max _{p_{2}} & \pi_{2} \\
\text { s.t. } & p_{2}>2 w_{2}-c . \\
& \frac{a+w_{2}-\sqrt{\left(a-w_{2}\right)^{2}-4 \hat{\pi}_{1}(z)}}{2}<p_{2}<\frac{a+w_{2}+\sqrt{\left(a-w_{2}\right)^{2}-4 \hat{\pi}_{1}(z)}}{2}
\end{array}
$$

The optimal solution for the unconstrained problem (B.10) is $p_{2}=\frac{a+w_{2}}{2}$. Constraint (B.11) is met when $w_{B}<\frac{a+2 c}{3}$; Constraint (B.12) is always met for $w_{2} \leq a-2 \sqrt{\hat{\pi}_{1}(z)}$. Therefore, the optimal retail price is $p_{2}=\frac{a+w_{2}}{2}$ for $w_{2}<\frac{a+2 c}{3}$.

When $w_{2} \geq \frac{a+2 c}{3}$, (B.11) is violated, and the candidate solution is $p_{2}=2 w_{2}-c$. Note that when $w_{2}=\frac{a+2 c}{3}$, we have $\frac{a+w_{2}-\sqrt{\left(a-w_{2}\right)^{2}-4 \hat{\pi}_{1}(z)}}{2}<\frac{a+w_{2}}{2}=2 w_{2}-c<\frac{a+w_{2}+\sqrt{\left(a-w_{2}\right)^{2}-4 \hat{\pi}_{1}(z)}}{2}$. Moreover, $\frac{a+w_{2}-\sqrt{\left(a-w_{2}\right)^{2}-4 \hat{\pi}_{1}(z)}}{2}<2 w_{2}-c<\frac{a+w_{2}+\sqrt{\left(a-w_{2}\right)^{2}-4 \hat{\pi}_{1}(z)}}{2}$ holds when $\frac{a+3 c-\sqrt{(a-c)^{2}-8 \hat{\pi}_{1}(z)}}{4}<w_{2}<$ $\frac{a+3 c+\sqrt{(a-c)^{2}-8 \hat{\pi}_{1}(z)}}{4}$. Since $\frac{a+3 c-{\sqrt{(a-c)^{2}-8 \hat{\pi}_{1}(z)}}_{4}}{4}<\frac{a+2 c}{3}$, the best response is $p_{2}=2 w_{2}-c$ for $\frac{a+2}{3} \leq$ $w_{2}<\frac{a+3 c+\sqrt{(a-c)^{2}-8 \hat{\pi}_{1}(z)}}{4}$. When $w_{2} \geq \frac{a+3 c+\sqrt{(a-c)^{2}-8 \hat{\pi}_{1}(z)}}{4}$, there is no feasible region for (B.10). Therefore, the optimal retail price is given by

$$
p_{2}= \begin{cases}\text { Infeasible, } & \text { if } w_{2} \geq \max \left\{\frac{a+3 c+\sqrt{(a-c)^{2}-8 \hat{\pi}_{1}(z)}}{4}, \frac{a+2 c}{3}\right\} \\ 2 w_{2}-c, & \text { if } \frac{a+2 c}{3} \leq w_{2}<\max \left\{\frac{a+3 c+\sqrt{(a-c)^{2}-8 \hat{\pi}_{1}(z)}}{4}, \frac{a+2 c}{3}\right\} \\ \frac{a+w_{2}}{2}, & \text { if } w_{2}<\frac{a+2 c}{3}\end{cases}
$$


Case 2. Retailer 2 is behind Retailer 1 but not behind the supplier, or equivalently, (1) $\left(a-p_{2}\right) \cdot\left(p_{2}-\right.$ $\left.w_{2}\right) \leq \hat{\pi}_{1}(z)$, i.e., $p_{2} \leq \frac{a+w_{2}-\sqrt{\left(a-w_{2}\right)^{2}-4 \hat{\pi}_{1}(z)}}{2}$, or $p_{2} \geq \frac{a+w_{2}+\sqrt{\left(a-w_{2}\right)^{2}-4 \hat{\pi}_{1}(z)}}{2} ;(2)\left(a-p_{2}\right) \cdot\left(p_{2}-w_{2}\right)>$ $\left(a-p_{2}\right)\left(w_{2}-c\right)$, i.e., $p_{2}>2 w_{2}-c$. then Retailer 2's problem is given by

$$
\begin{array}{ll}
\max _{p_{2}} & \pi_{2}-\hat{p}(z) \cdot \rho \cdot\left(\hat{\pi}_{1}(z)-\pi_{2}\right) \\
\text { s.t. } & p_{2}>2 w_{2}-c . \\
& p_{2} \geq \frac{a+w_{2}+\sqrt{\left(a-w_{2}\right)^{2}-4 \hat{\pi}_{1}(z)}}{2} \text { or } p_{2} \leq \frac{a+w_{2}-\sqrt{\left(a-w_{2}\right)^{2}-4 \hat{\pi}_{1}(z)}}{2}
\end{array}
$$

The optimal solution for the unconstrained problem (B.14) is $p_{2}=\frac{a+w_{2}}{2}$. For this response, constraint (B.15) is met when $w_{2}<\frac{a+2 c}{3}$; Constraint (B.16) is not met for $w_{2}<a-2 \sqrt{\hat{\pi}_{1}(z}$ ). However, when $w_{2}<\frac{a+2 c}{3}$, it is not optimal for Retailer 2 to meet constraint (B.16) because Retailer 2 can achieve a higher utility when (B.16) is not met. Note the objective function (B.14) is equivalent to $(1+\hat{p}(z) \cdot \rho) \pi_{2}-\hat{p}(z) \cdot \rho \cdot \hat{\pi}_{1}(z)$, and $\hat{p}(z) \cdot \rho \cdot \hat{\pi}_{1}(z)$ is a constant. The optimal solution to $\pi_{2}$, when feasible, is also optimal to the current problem (B.14). Therefore, $p_{2}=\frac{a+w_{2}}{2}$ is the optimal retail price for $w_{2}<\frac{a+2 c}{3}$.

When $w_{2} \geq \frac{a+2 c}{3}$, (B.15) is violated for the unconstrained optimal solution and the candidate solution is $p_{2}=2 w_{2}-c$. (B.16) holds when $w_{2} \geq \frac{a+3 c+\sqrt{(a-c)^{2}-8 \hat{\pi}_{1}(z)}}{4}$ (i.e., the first inequality of (B.16) holds) or $w_{2} \leq \frac{a+3 c-\sqrt{(a-c)^{2}-8 \hat{\pi}_{1}(z)}}{4}$ (i.e., the second inequality of (B.16) holds). Note here $\frac{a+3 c-\sqrt{(a-c)^{2}-8 \hat{\pi}_{1}(z)}}{4}<\frac{a+2 c}{3}$. When $w_{2} \leq \frac{a+3 c+\sqrt{(a-c)^{2}-8 \hat{\pi}_{1}(z)}}{4}, p_{2}=\frac{a+w_{2}+\sqrt{\left(a-w_{2}\right)^{2}-4 \hat{\pi}_{1}(z)}}{2}$ and (B.15) holds.

Therefore, the optimal retail price is given by

$$
p_{2}= \begin{cases}2 w_{2}-c, & \text { if } w_{2} \geq \max \left\{\frac{a+3 c+\sqrt{(a-c)^{2}-8 \hat{\pi}_{1}(z)}}{4}, \frac{a+2 c}{3}\right\} \\ \frac{a+w_{2}+\sqrt{\left(a-w_{2}\right)^{2}-4 \hat{\pi}_{1}(z)}}{2}, & \text { if } \frac{a+2 c}{3} \leq w_{2}<\max \left\{\frac{a+3 c+\sqrt{(a-c)^{2}-8 \hat{\pi}_{1}(z)}}{4}, \frac{a+2 c}{3}\right\} \\ \frac{a+w_{2}}{2}, & \text { if } w_{2}<\frac{a+2 c}{3}\end{cases}
$$

Combining Cases 1 and 2: In Cases 1 and 2, Retailer 2 does not experience distributional fairness (i.e., $p_{2}>2 w_{2}-c$ ). For $w_{2} \geq \max \left\{\frac{a+3 c+\sqrt{(a-c)^{2}-8 \hat{\pi}_{1}(z)}}{4}, \frac{a+2 c}{3}\right\}$, the only feasible best response is from Case 2. For $\frac{a+2 c}{3} \leq w_{2}<\max \left\{\frac{a+3 c+\sqrt{(a-c)^{2}-8 \hat{\pi}_{1}(z)}}{4}, \frac{a+2 c}{3}\right\}$, Cases 1 and 2 have give different best responses. However, the overall best response in this region is $p_{2}=2 w_{2}-c$ for the following reasons: In case 1, Retailer 2's total utility is $u_{2}^{1}=\pi_{2}\left(2 w_{2}-c\right)$, and in case 2, $u_{2}^{2}=\pi_{2}\left(\frac{a+w_{2}+\sqrt{\left(a-w_{2}\right)^{2}-4 \hat{\pi}_{1}(z)}}{2}\right)$. In this region, we also have $2 w_{2}-c \leq \frac{a+w_{2}+\sqrt{\left(a-w_{2}\right)^{2}-4 \hat{\pi}_{1}(z)}}{2}$. Furthermore, the optimal solution for $\pi_{2}$ without any constraint is $\frac{a+w_{2}}{2}$. When $w_{2} \geq \frac{a+2 c}{3}$, we have $\frac{a+w_{2}}{2} \leq 2 w_{2}-c$. Because $\pi_{2}$ is concave in $p_{2}$, we have $\pi_{2}\left(2 w_{2}-c\right) \geq \pi_{2}\left(\frac{a+w_{2}+\sqrt{\left(a-w_{2}\right)^{2}-4 \hat{\pi}_{1}(z)}}{2}\right)$, that is $u_{2}^{1} \geq u_{2}^{2}$. Therefore, $2 w_{2}-c$ is the best response for $\frac{a+2 c}{3} \leq w_{2}<\max \left\{\frac{a+3 c+\sqrt{(a-c)^{2}-8 \hat{\pi}_{1}(z)}}{4}, \frac{a+2 c}{3}\right\}$. When $w_{2}<\frac{a+2 c}{3}$, both cases have the same best response. 
Combining the two best responses (B.13) and (B.17), we have the following optimal retail price for the case wherein Retailer 2 does not experience distributional fairness

$$
p_{2}= \begin{cases}2 w_{2}-c, & \text { if } w_{2} \geq \frac{a+2 c}{3} \\ \frac{a+w_{2}}{2}, & \text { if } w_{2}<\frac{a+2 c}{3}\end{cases}
$$

Case 3. Retailer 2 is behind both Retailer 1 and the supplier, or equivalently, (1) $\left(a-p_{2}\right) \cdot\left(p_{2}-w_{2}\right) \leq$ $\hat{\pi}_{1}(z)$, i.e., $p_{2} \geq \frac{a+w_{2}+\sqrt{\left(a-w_{2}\right)^{2}-4 \hat{\pi}_{1}(z)}}{2}$ or $p_{2} \leq \frac{a+w_{2}-\sqrt{\left(a-w_{2}\right)^{2}-4 \hat{\pi}_{1}(z)}}{2} ;(2)\left(a-p_{2}\right) \cdot\left(p_{2}-w_{2}\right) \leq(a-$ $\left.p_{2}\right) \cdot\left(w_{2}-c\right)$, i.e., $p_{2} \leq 2 w_{2}-c$, then Retailer 2's problem is given by

$$
\begin{array}{ll}
\max _{p_{2}} & \pi_{2}-\delta \cdot\left(\pi_{S, 2}-\pi_{2}\right)-\hat{p}(z) \cdot \rho \cdot\left(\hat{\pi}_{1}(z)-\pi_{2}\right) \\
\text { s.t. } & p_{2} \leq 2 w_{2}-c, \\
& p_{2} \geq \frac{a+w_{2}+\sqrt{\left(a-w_{2}\right)^{2}-4 \hat{\pi}_{1}(z)}}{2} \text { or } p_{2} \leq \frac{a+w_{2}-\sqrt{\left(a-w_{2}\right)^{2}-4 \hat{\pi}_{1}(z)}}{2}
\end{array}
$$

The optimal solution for the unconstrained problem (B.19) is $p_{2}=\frac{a+w_{2}}{2}+\frac{\delta\left(w_{2}-c\right)}{2(1+\delta+\hat{p}(z) \cdot \rho)}$. Constraint (B.20) is met when $w_{2} \geq w_{2}^{I}=\frac{a(1+\delta+\hat{p}(z) \cdot \rho)+c(2(1+\hat{p}(z) \cdot \rho)+\delta)}{3(1+\hat{p}(z) \cdot \rho)+2 \delta}$; Constraint (B.21) is met when $w_{2} \geq w_{2}^{I I}:=$ $\frac{a-\psi \cdot c-\sqrt{\psi(a-c)^{2}+(1-\psi) 4 \hat{\pi}_{1}(z)}}{1-\psi}$. Here, $\psi:=\left(\frac{\delta}{1+\delta+\hat{p}(z) \cdot \rho}\right)^{2}$.

Note in Case 3 , we only need to consider $w_{2} \geq \frac{a+2 c}{3}$. For $w_{2}<\frac{a+2 c}{3}$, the objective function in Case 2 is superior and is achievable by choosing $p_{2}=\frac{a+w_{2}}{2}$.

When $w_{2}^{I I}>w_{2}^{I}$ and $w_{2} \geq w_{2}^{I I}$, the optimal retail price is $p_{2}=\frac{a+w_{2}}{2}+\frac{\delta\left(w_{2}-c\right)}{2(1+\delta+\hat{p}(z) \cdot \rho)}$. When $w_{2}<w_{2}^{I I}$, (B.21) is violated, and the candidate solution is $p_{2}=\frac{a+w_{2}+\sqrt{\left(a-w_{2}\right)^{2}-4 \hat{\pi}_{1}(z)}}{2}$. Note that when $w_{2}=w_{2}^{I I}$, we have $p_{2}=\frac{a+w_{2}}{2}+\frac{\delta\left(w_{2}-c\right)}{2(1+\delta+\hat{p}(z) \cdot \rho)}=\frac{a+w_{2}+\sqrt{\left(a-w_{2}\right)^{2}-4 \hat{\pi}_{1}(z)}}{2} \leq 2 w_{2}-c$. The inequality holds because $w_{2}^{I I}>w_{2}^{I}$. Moreover, this inequality holds for $\frac{a+3 c+\sqrt{(a-c)^{2}-8 \hat{\pi}_{1}(z)}}{4} \leq w_{2}<w_{2}^{I I}{ }^{14}$ Therefore, the optimal retail price is $p_{2}=\frac{a+w_{2}+\sqrt{\left(a-w_{2}\right)^{2}-4 \hat{\pi}_{1}(z)}}{2}$ for $\frac{a+3 c+\sqrt{(a-c)^{2}-8 \hat{\pi}_{1}(z)}}{4} \leq w_{2}<w_{2}^{I I}$. When $\frac{a+3 c-\sqrt{(a-c)^{2}-8 \hat{\pi}_{1}(z)}}{4}<w_{2}<\frac{a+3 c+\sqrt{(a-c)^{2}-8 \hat{\pi}_{1}(z)}}{4}$, (B.20) and (B.21) give $\frac{a+w_{2}-\sqrt{\left(a-w_{2}\right)^{2}-4 \hat{\pi}_{1}(z)}}{2}$ as the only feasible solution. Note here $\frac{a+3 c-\sqrt{(a-c)^{2}-8 \hat{\pi}_{1}(z)}}{4}<\frac{a+2 c}{3}$. Therefore, for $\frac{a+2 c}{3} \leq w_{2}<$ $\frac{a+3 c+\sqrt{(a-c)^{2}-8 \hat{\pi}_{1}(z)}}{4}$, the best response $p_{2}=\frac{a+w_{2}-\sqrt{\left(a-w_{2}\right)^{2}-4 \hat{\pi}_{1}(z)}}{2}$.

Therefore, the optimal retail price for $w_{2}^{I I}>w_{2}^{I}$ is given by

$$
p_{2}= \begin{cases}\frac{a+w_{2}}{2}+\frac{\delta\left(w_{2}-c\right)}{2(1+\delta+\hat{p}(z) \cdot \rho)}, & \text { if } w_{2} \geq w_{2}^{I I} \\ \frac{a+w_{2}+\sqrt{\left(a-w_{2}\right)^{2}-4 \hat{\pi}_{1}\left(w_{1}\right)}}{2}, & \text { if } \frac{a+3 c+\sqrt{(a-c)^{2}-8 \hat{\pi}_{1}(z)}}{4} \leq w_{2}<w_{2}^{I I} \\ \frac{a+w_{2}-\sqrt{\left(a-w_{2}\right)^{2}-4 \hat{\pi}_{1}(z)}}{2}, & \text { if } \frac{a+2 c}{3} \leq w_{2}<\frac{a+3 c+\sqrt{(a-c)^{2}-8 \hat{\pi}_{1}(z)}}{4}\end{cases}
$$

When $w_{2}^{I I} \leq w_{2}^{I}$ and $w_{2}>w_{2}^{I}$, the optimal retail price is $p_{2}=\frac{a+w_{2}}{2}+\frac{\delta\left(w_{2}-c\right)}{2(1+\delta+\hat{p}(z) \cdot \rho)}$. When $w_{2}<w_{2}^{I}$, (B.20) is not met, and the candidate solution is $p_{2}=2 w_{2}-c$. Note that when $w_{2}=w_{2}^{I}$, we

\footnotetext{
${ }^{14}$ Since we only consider $w_{2} \geq \frac{a+2 c}{3}$ and $w_{2}<a-2 \sqrt{\hat{\pi}_{1}(z)}$, these two conditions imply that $\hat{\pi}_{1}(z)<$ $\frac{(a-c)^{2}}{9}$ and thus $(a-c)^{2}-8 \hat{\pi}_{1}(z)>0$.
} 
have $p_{2}=\frac{a+w_{2}}{2}+\frac{\delta\left(w_{2}-c\right)}{2(1+\delta+\hat{p}(z) \cdot \rho)}=2 w_{2}-c \geq \frac{a+w_{2}+\sqrt{\left(a-w_{2}\right)^{2}-4 \hat{\pi}_{1}(z)}}{2}$. The inequality holds because $w_{2}^{I}>w_{2}^{I I}$. Moreover, this inequality holds for $\frac{a+3 c+\sqrt{(a-c)^{2}-8 \hat{\pi}_{1}(z)}}{4} \leq w_{2}<w_{2}^{I}$. Therefore, the best response is $p_{2}=2 w_{2}-c$ for $\frac{a+3 c+\sqrt{(a-c)^{2}-8 \hat{\pi}_{1}(z)}}{4} \leq w_{2}<w_{2}^{I}$. When $\frac{a+3 c-\sqrt{(a-c)^{2}-8 \hat{\pi}_{1}(z)}}{4}<w_{2}<$ $\frac{a+3 c+\sqrt{(a-c)^{2}-8 \hat{\pi}_{1}(z)}}{4},\left(\right.$ B.20) and (B.21) give $\frac{a+w_{2}-\sqrt{\left(a-w_{2}\right)^{2}-4 \hat{\pi}_{1}(z)}}{2}$ as the only feasible solution. Note here $\frac{a+3 c-\sqrt{(a-c)^{2}-8 \hat{\pi}_{1}(z)}}{4}<\frac{a+2 c}{3}$. Therefore, for $\frac{a+2 c}{3} \leq w_{2}<\frac{a+3 c-\sqrt{(a-c)^{2}-8 \hat{\pi}_{1}(z)}}{4}$, the optimal retail price $p_{2}=\frac{a+w_{2}-\sqrt{\left(a-w_{2}\right)^{2}-4 \hat{\pi}_{1}(z)}}{2}$.

The optimal retail price for $w_{2}^{I I} \leq w_{2}^{I}$ is given by

$$
p_{2}= \begin{cases}\frac{a+w_{2}}{2}+\frac{\delta\left(w_{2}-c\right)}{2(1+\delta+\hat{p}(z) \cdot \rho))}, & \text { if } w_{2} \geq w_{2}^{I} \\ 2 w_{2}-c, & \text { if } \frac{a+3 c+\sqrt{(a-c)^{2}-8 \hat{\pi}_{1}(z)}}{4} \leq w_{2}<w_{2}^{I} \\ \frac{a+w_{2}-\sqrt{\left(a-w_{2}\right)^{2}-4 \hat{\pi}_{1}(z)}}{2}, & \text { if } \frac{a+2 c}{3} \leq w_{2}<\frac{a+3 c+\sqrt{(a-c)^{2}-8 \hat{\pi}_{1}(z)}}{4}\end{cases}
$$

Case 4. When Retailer 2 is behind the supplier but not behind Retailer 1 , that is, $(1)\left(a-p_{2}\right) \cdot\left(p_{2}-w_{2}\right)>$ $\hat{\pi}_{1}(z)$, i.e., $\frac{a+w_{2}-\sqrt{\left(a-w_{2}\right)^{2}-4 \hat{\pi}_{1}(z)}}{2}<p_{2}<\frac{a+w_{2}-\sqrt{\left(a-w_{2}\right)^{2}-4 \hat{\pi}_{1}(z)}}{2} ;(2)\left(a-p_{2}\right) \cdot\left(p_{2}-w_{2}\right) \leq\left(a-p_{2}\right) \cdot\left(w_{2}-c\right)$, i.e., $p_{2} \leq 2 w_{2}-c$, then Retailer 2 's problem is given by

$$
\begin{array}{ll}
\max _{p_{2}} & \pi_{2}-\delta \cdot\left(\pi_{S, 2}-\pi_{2}\right) \\
\text { s.t. } & p_{2} \leq 2 w_{2}-c . \\
& \frac{a+w_{2}-\sqrt{\left(a-w_{2}\right)^{2}-4 \hat{\pi}_{1}(z)}}{2}<p_{2}<\frac{a+w_{2}+\sqrt{\left(a-w_{2}\right)^{2}-4 \hat{\pi}_{1}(z)}}{2}
\end{array}
$$

The optimal solution for the unconstrained problem (B.24) is $p_{2}=\frac{a+w_{2}}{2}+\frac{\delta\left(w_{2}-c\right)}{2(1+\delta)}$. Constraint (B.25) is met when $w_{2} \geq \frac{a(1+\delta)+c(2+\delta)}{3+2 \delta}$; Constraint (B.26) is met when $w_{2}<w_{2}^{I I I}:=\frac{a-\phi c-\sqrt{\phi(a-c)^{2}+(1-\phi) 4 \hat{\pi}_{1}(z)}}{1-\phi}$. Here, $\phi:=\left(\frac{\delta}{1+\delta}\right)^{2}$. Note here $w_{2}^{I I I} \leq w_{2}^{I I}$.

Note similar to Case 3, we only need to consider $w_{2} \geq \frac{a+2 c}{3}$. For $w_{2}<\frac{a+2 c}{3}$, the objective function in Case 1 is superior and is achievable by choosing $p_{2}=\frac{a+w_{2}}{2}$.

When $w_{2}^{I I I}>\frac{a(1+\delta)+c(2+\delta)}{3+2 \delta}$ and $\frac{a(1+\delta)+c(2+\delta)}{3+2 \delta} \leq w_{B}<w_{3}^{I I I}$, the optimal retail price is $p_{2}=\frac{a+w_{2}}{2}+$ $\frac{\delta\left(w_{2}-c\right)}{2(1+\delta)}$. When $w_{2} \geq w_{2}^{I I I},\left(\right.$ B.26) is violated, and the candidate solution is $p_{2}=\frac{a+w_{2}+\sqrt{\left(a-w_{2}\right)^{2}-4 \hat{\pi}_{1}(z)}}{2}$. Note that when $w_{2}=w_{2}^{I I I}$, we have $p_{2}=\frac{a+w_{2}}{2}+\frac{\delta\left(w_{2}-c\right)}{2(1+\delta)}=\frac{a+w_{2}+\sqrt{\left(a-w_{2}\right)^{2}-4 \hat{\pi}_{1}(z)}}{2} \leq 2 w_{2}-c$. The inequality $\frac{a+w_{2}+\sqrt{\left(a-w_{2}\right)^{2}-4 \hat{\pi}_{1}(z)}}{2} \leq 2 w_{2}-c$ always holds for $w_{2} \geq w_{2}^{I I I}$ because $F\left(w_{2}\right):=2 w_{2}-$ $c-\left(\frac{a+w_{2}+\sqrt{\left(a-w_{2}\right)^{2}-4 \hat{\pi}_{1}(z)}}{2}\right)$ is increasing in $w_{2}$. Note here $w_{2}^{I I I}>\frac{a+3 c+\sqrt{(a-c)^{2}-8 \hat{\pi}_{1}(z)}}{4}$, because $F\left(\frac{a+3 c+\sqrt{(a-c)^{2}-8 \hat{\pi}_{1}(z)}}{4}\right)=0$.

For $w_{2}<\frac{a(1+\delta)+c(2+\delta)}{3+2 \delta},\left(\right.$ B.25) is violated, and the candidate solution is $p_{2}=2 w_{2}-c$. Note when $w_{2}=\frac{a(1+\delta)+c(2+\delta)}{3+2 \delta}$, we have $p_{2}=\frac{a+w_{2}}{2}+\frac{\delta\left(w_{2}-c\right)}{2(1+\delta)}=2 w_{2}-c<\frac{a+w_{2}+\sqrt{\left(a-w_{2}\right)^{2}-4 \hat{\pi}_{1}(z)}}{2}$. Then $2 w_{2}-c<$ 
$\frac{a+w_{2}+\sqrt{\left(a-w_{2}\right)^{2}-4 \hat{\pi}_{1}(z)}}{2}$ holds for all $w_{2}<\frac{a(1+\delta)+c(2+\delta)}{3+2 \delta}$ because $F\left(w_{2}\right)$ is increasing in $w_{2}$. When $p_{2}=2 w_{2}-c, \frac{a+w_{2}-\sqrt{\left(a-w_{2}\right)^{2}-4 \hat{\pi}_{1}(z)}}{2}<p_{2}$ in (B.26) holds when $w_{2}>\frac{a+3 c-\sqrt{(a-c)^{2}-8 \hat{\pi}_{1}(z)}}{4}$. Note here $\frac{a+3 c-\sqrt{(a-c)^{2}-8 \hat{\pi}_{1}(z)}}{4}<\frac{a+2 c}{3}$. Therefore, for $\frac{a+2 c}{3} \leq w_{2}<\frac{a(1+\delta)+c(2+\delta)}{3+2 \delta}$, the optimal retail price is $p_{2}=2 w_{2}-c$.

Therefore, the optimal response when $w_{2}^{I I I}>\frac{a(1+\delta)+c(2+\delta)}{3+2 \delta}$ is given by

$$
p_{2}= \begin{cases}\frac{a+w_{2}+\sqrt{\left(a-w_{2}\right)^{2}-4 \hat{\pi}_{1}(z)}}{2}, & \text { if } w_{2} \geq w_{2}^{I I I} \\ \frac{a+w_{2}}{2}+\frac{\delta\left(w_{2}-c\right)}{2(1+\delta)}, & \text { if } \frac{a(1+\delta)+c(2+\delta)}{3+2 \delta} \leq w_{2}<w_{2}^{I I I} \\ 2 w_{2}-c, & \text { if } \frac{a+2 c}{3} \leq w_{2}<\frac{a(1+\delta)+c(2+\delta)}{3+2 \delta}\end{cases}
$$

When $w_{2}^{I I I} \leq \frac{a(1+\delta)+c(2+\delta)}{3+2 \delta}$, the unconstrained optimal solution is not feasible. Then the optimal retail price occurs on one of the binding constraint, and is given by

$$
p_{2}= \begin{cases}\frac{a+w_{2}+\sqrt{\left(a-w_{2}\right)^{2}-4 \hat{\pi}_{1}(z)}}{2}, & \text { if } w_{2} \geq \frac{a+3 c+\sqrt{(a-c)^{2}-8 \hat{\pi}_{1}(z)}}{4} \\ 2 w_{2}-c, & \text { if } \frac{a+2 c}{3} \leq w_{2}<\frac{a+3 c+\sqrt{(a-c)^{2}-8 \hat{\pi}_{1}(z)}}{4}\end{cases}
$$

Combining Cases 3 and 4, and with 1 and 2 to obtain the best-response retail price for the entire problem In cases 3 and 4, Retailer 2 experiences distributional fairness. The two cases are distinguished by whether Retailer 2 experiences peer-induced fairness. The two cases can be "pasted" together to obtain the best responses when distributional fairness is salient. Cases 1 and 2 have been combined and can then be added to obtain the best response of the entire problem. However, both case 3 and 4 have two possible response functions, and in total there are four possible optimal responses as follows.

\section{Best-Response Retail Price 1.}

When $w_{2}^{I I}>w_{2}^{I}$ and $w_{2}^{I I I}>\frac{a(1+\delta)+c(2+\delta)}{3+2 \delta}$, we need to combine (B.22), (B.27) and (B.18), and the optimal response is given as follows

$$
p_{2}^{* *}= \begin{cases}\frac{a+w_{2}}{2}+\frac{\delta\left(w_{2}-c\right)}{2(1+\delta+\hat{p}(z) \cdot \rho)}, & \text { if } w_{2} \geq w_{2}^{I I} \\ \frac{a+w_{2}+\sqrt{\left(a-w_{2}\right)^{2}-4 \hat{\pi}_{1}(z)}}{2}, & \text { if } w_{2}^{I I I} \leq w_{2}<w_{2}^{I I} \\ \frac{a+w_{2}}{2}+\frac{\delta\left(w_{2}-c\right)}{2(1+\delta)}, & \text { if } \frac{a(1+\delta)+c(2+\delta)}{3+2 \delta} \leq w_{2}<w_{2}^{I I I} \\ 2 w_{2}-c, & \text { if } \frac{a+2 c}{3} \leq w_{2}<\frac{a(1+\delta)+c(2+\delta)}{3+2 \delta} \\ \frac{a+w_{2}}{2}, & \text { if } w_{2}<\frac{a+2 c}{3}\end{cases}
$$

This response function takes the optimal retail price from Case 4 from $w_{2}<w_{2}^{I I I}$ and from Case 3 for $w_{2} \geq w_{2}^{I I I}$. For $\frac{a(1+\delta)+c(2+\delta)}{3+2 \delta} \leq w_{2}<w_{2}^{I I I}$, Case 4 has an interior optimal solution $p_{2}=\frac{a+w_{2}}{2}+\frac{\delta\left(w_{2}-c\right)}{2(1+\delta)}$ and the objective function is alway superior to the one in Case 3. Therefore, $\frac{a+w_{2}}{2}+\frac{\delta\left(w_{2}-c\right)}{2(1+\delta)}$ is the best response for Case 3 and 4 combined when $\frac{a(1+\delta)+c(2+\delta)}{3+2 \delta} \leq w_{2}<w_{2}^{I I I}$. For $\frac{a+2 c}{3} \leq w_{2}<\frac{a(1+\delta)+c(2+\delta)}{3+2 \delta}$, we compare the values of the objective functions in Cases 3 and 4 as follows: the utility in Case 3 is 
$u_{2}^{3}=u\left(\frac{a+w_{2}-\sqrt{\left(a-w_{2}\right)^{2}-4 \hat{\pi}_{1}(z)}}{2}\right)$, and in Case 4, $u_{2}^{4}=u\left(2 w_{2}-c\right)$, where $u\left(p_{2}\right):=\pi_{2}-\delta \cdot\left(\pi_{S, 2}-\pi_{2}\right)$ is concave in $p_{2}$. Note that when $\frac{a+2 c}{3} \leq w_{2}<\frac{a(1+\delta)+c(2+\delta)}{3+2 \delta}$, we have $\frac{a+w_{2}}{2}+\frac{\delta\left(w_{2}-c\right)}{2(1+\delta)}>2 w_{2}-c>$ $\frac{a+w_{2}-\sqrt{\left(a-w_{2}\right)^{2}-4 \hat{\pi}_{1}(z)}}{2}$. Since $p_{2}=\frac{a+w_{2}}{2}+\frac{\delta\left(w_{2}-c\right)}{2(1+\delta)}$ maximizes $u\left(p_{2}\right)$ and $u$ is concave, we have $u_{2}^{4} \geq u_{2}^{3}$, and $p_{2}=2 w_{2}-c$ is optimal for $\frac{a+2 c}{3} \leq w_{2}<\frac{a(1+\delta)+c(2+\delta)}{3+2 \delta}$. Recall that $w_{2}^{I I I} \leq w_{2}^{I I}$, and the best responses for $w_{2}^{I I I} \leq w_{2}<w_{2}^{I I}$ are common in both cases (Also recall that $w_{2}^{I I I}>\frac{a+3 c+\sqrt{(a-c)^{2}-8 \hat{\pi}_{1}(z)}}{4}$ ). When $w_{2} \geq w_{2}^{I I}, \frac{a+w_{2}}{2}+\frac{\delta\left(w_{2}-c\right)}{2(1+\delta+\hat{p}(z) \cdot \rho)}$ is the interior optimal solution to Case 3 , and it is optimal for Case 3 and 4 combined because the best response in Case 4 is on the boundary of the feasible region of Case 3 and thus is suboptimal to Case 3. The last line in the response function is pasted from (B.18).

\section{Supplier's Pricing Decision Under Best-Response Retail Price 1}

Given the best response function (B.29), the optimal wholesale price offer can be found as follows: (1) When $w_{2} \geq w_{2}^{I I}$, the supplier's profit function is $\pi_{S, 2}=\left(a-\frac{a+w_{2}}{2}-\frac{\delta\left(w_{2}-c\right)}{2(1+\delta+\hat{p}(z) \cdot \rho)}\right) \cdot\left(w_{2}-c\right)$. The optimal solution is $w_{2}=\frac{a+c}{2}-\frac{\delta(a-c)}{2(1+2 \delta+\hat{p}(z) \cdot \rho)}$. The solution satisfies the condition $w_{2} \geq w_{2}^{I I}$ when $\hat{\pi}_{1}(z) \geq \frac{(a-c)^{2}(1+4 \delta+\hat{p}(z) \cdot \rho)}{16(1+2 \delta+\hat{p}(z) \cdot \rho)}$. When $\hat{\pi}_{1}(z)<\frac{(a-c)^{2}(1+4 \delta+\hat{p}(z) \cdot \rho)}{16(1+2 \delta+\hat{p}(z) \cdot \rho)}$, the optimal decision is $w_{2}=w_{2}^{I I} ;(2)$ When $w_{2}^{I I I} \leq w_{2}<w_{2}^{I I}$, the supplier's profit is $\pi_{S, 2}=\left(a-\frac{a+w_{2}+\sqrt{\left(a-w_{2}\right)^{2}-4 \hat{\pi}_{1}(z)}}{2}\right) \cdot\left(w_{2}-c\right)$ that is an increasing function of $w_{2}$. Therefore, the optimal solution is $w_{2}=w_{B}^{I I} ;(3)$ When $\frac{a(1+\delta)+c(2+\delta)}{3+2 \delta} \leq$ $w_{2}<w_{2}^{I I I}$, the supplier's profit is $\pi_{S, 2}=\left(a-\frac{a+w_{2}}{2}-\frac{\delta\left(w_{2}-c\right)}{2(1+\delta)}\right)\left(w_{2}-c\right)$. The optimal solution is $w_{2}=\frac{a+c}{2}-\frac{\delta(a-c)}{2(1+2 \delta)}$ that satisfies $\frac{a(1+\delta)+c(2+\delta)}{3+2 \delta} \leq w_{2}<w_{2}^{I I I}$ when $\delta<\frac{1}{7}$ and $\hat{\pi}_{1}(z)<\frac{(a-c)^{2}(1+4 \delta)}{16(1+2 \delta)} ;(4)$ When $\frac{a+2 c}{3} \leq w_{2}<\frac{a(1+\delta)+c(2+\delta)}{3+2 \delta}$, the supplier's profit is $\pi_{S, 2}=\left(a-2 w_{2}+c\right)\left(w_{2}-c\right)$. The optimal solution is $w_{2}=\frac{a+3 c}{4}$ that is not within the boundaries of $\frac{a+2 c}{3} \leq w_{2}<\frac{a(1+\delta)+c(2+\delta)}{3+2 \delta}$, and the optimal wholesale price is $w_{2}=\frac{a+2 c}{3} ;(5)$ When $w_{2}<\frac{a+2 c}{3}$, the supplier's profit is $\pi_{S, 2}=\left(a-\frac{a+w_{2}}{2}\right)\left(w_{2}-c\right)$. The optimal solution is $w_{2}=\frac{a+c}{2}$ that is not within the boundaries of $w_{2}<\frac{a+2 c}{3}$, and the optimal wholesale price is $w_{2}=\frac{a+2 c}{3}$.

For (1), the supplier's profit is $\frac{(a-c)^{2}(1+\delta+\hat{p}(z) \cdot \rho)}{8(1+2 \delta+\hat{p}(z) \rho)}$ when $\hat{\pi}_{1}(z) \geq \frac{(a-c)^{2}(1+4 \delta+\hat{p}(z) \cdot \rho)}{16(1+2 \delta+\hat{p}(z) \cdot \rho)}$, for (2) the supplier's profit is $\pi_{S, 2}\left(w_{2}^{I I}\right)=\left(a-\frac{a+w_{2}^{I I}+\sqrt{\left(a-w_{2}^{I I}\right)^{2}-4 \hat{\pi}_{1}(z)}}{2}\right) \cdot\left(w_{2}^{I I}-c\right)$ for $(3)$ the supplier's profit is $\frac{(a-c)^{2}(1+\delta)}{8(1+2 \delta)}$ and for $(4)$ and $(5)$, the supplier's profit is $\frac{(a-c)^{2}}{9}$.

Note that it always holds that $\frac{(a-c)^{2}(1+\delta+\hat{p}(z) \cdot \rho)}{8(1+2 \delta+\hat{p}(z) \rho)}>\frac{(a-c)^{2}(1+\delta)}{8(1+2 \delta)}$ for $\delta>0$. Furthermore, we have $\frac{(a-c)^{2}(1+\delta+\hat{p}(z) \cdot \rho)}{8(1+2 \delta+\hat{p}(z) \cdot \rho)}>\frac{(a-c)^{2}}{9}$ when $\delta<\frac{1+\hat{p}(z) \rho}{7}$, and $\frac{(a-c)^{2}(1+\delta)}{8(1+2 \delta)}>\frac{(a-c)^{2}}{9}$ when $\delta<\frac{1}{7}$.

When $\hat{\pi}_{1}(z) \geq \frac{(a-c)^{2}(1+4 \delta+\hat{p}(z) \cdot \rho)}{16(1+2 \delta+\hat{p}(z) \cdot \rho)}$, the supplier's optimal pricing decision is

$$
w_{2}^{* *}= \begin{cases}\frac{a+c}{2}-\frac{\delta(a-c)}{2(1+2 \delta+\hat{p}(z) \cdot \rho)}, & \text { if } \delta<\frac{1+\hat{p}(z) \cdot \rho}{7} \\ \frac{a+2 c}{3}, & \text { otherwise }\end{cases}
$$

When $\hat{\pi}_{1}(z)<\frac{(a-c)^{2}(1+4 \delta+\hat{p}(z) \cdot \rho)}{16(1+2 \delta+\hat{p}(z) \cdot \rho)}$, the supplier's optimal decision is either $w_{2}^{I I}$ (i.e., the local optimal 
decision for $w_{2} \geq w_{2}^{I I I}$ ) or the following one (this is the local optimal decision for $w_{2}<w_{2}^{I I I}$ ),

$$
w_{2}^{* *}= \begin{cases}\frac{a+c}{2}-\frac{\delta(a-c)}{2(1+2 \delta)}, & \text { if } \delta<\frac{1}{7} \\ \frac{a+2 c}{3}, & \text { otherwise }\end{cases}
$$

By comparing the profits of the two local maximizers, $w_{2}^{I I}$ is optimal globally if $\pi_{S, 2}\left(w_{2}^{I I}\right) \geq \pi_{S, 2}\left(w_{2}^{* *}\right)$; otherwise, $w_{2}^{* *}$ in (B.31) is optimal.

Following the similar approach, we obtain the best-response retail price and optimal wholesale prices for the other three cases as follows:

\section{Best-Response Retail Price 2.}

The best response for the entire problem for $w_{2}^{I I} \leq w_{2}^{I}$ and $w_{2}^{I I I}>\frac{a(1+\delta)+c(2+\delta)}{3+2 \delta}$ is given as as follows

$$
p_{2}^{* *}= \begin{cases}\frac{a+w_{2}}{2}+\frac{\delta\left(w_{2}-c\right)}{2(1+\delta+\hat{p}(z) \cdot \rho)}, & \text { if } w_{2} \geq w_{2}^{I} \\ 2 w_{2}-c, & \text { if } w_{2}^{I I I} \leq w_{2}<w_{2}^{I} \\ \frac{a+w_{2}}{2}+\frac{\delta\left(w_{2}-c\right)}{2(1+\delta)}, & \text { if } \frac{a(1+\delta)+c(2+\delta)}{3+2 \delta} \leq w_{2}<w_{2}^{I I I} \\ 2 w_{2}-c, & \text { if } \frac{a+2 c}{3} \leq w_{2}<\frac{a(1+\delta)+c(2+\delta)}{3+2 \delta} \\ \frac{a+w_{2}}{2}, & \text { if } w_{2}<\frac{a+2 c}{3}\end{cases}
$$

The supplier's optimal decision is

$$
w_{2}^{* *}= \begin{cases}\frac{a+c}{2}-\frac{\delta(a-c)}{2(1+2 \delta+\hat{p}(z) \cdot \rho)}, & \text { if } \delta<\frac{1+\hat{p}(z) \cdot \rho}{7} \\ \frac{a+2 c}{3}, & \text { otherwise }\end{cases}
$$

\section{Best-Response Retail Price 3.}

The best-response retail price for the entire problem for $w_{2}^{I I}>w_{2}^{I}$ and $w_{2}^{I I I} \leq \frac{a(1+\delta)+c(2+\delta)}{3+2 \delta}$ is given as as follows

$$
p_{2}^{* *}= \begin{cases}\frac{a+w_{2}}{2}+\frac{\delta\left(w_{2}-c\right)}{2(1+\delta+\hat{p}(z) \cdot \rho)}, & \text { if } w_{2} \geq w_{2}^{I I} \\ \frac{a+w_{2}+\sqrt{\left(a-w_{2}\right)^{2}-4 \hat{\pi}_{1}(z)}}{2}, & \text { if } \frac{a+3 c+\sqrt{(a-c)^{2}-8 \hat{\pi}_{1}(z)}}{4} \leq w_{2}<w_{2}^{I I} \\ 2 w_{2}-c, & \text { if } \frac{a+2 c}{3} \leq w_{2}<\frac{a+3 c+\sqrt{(a-c)^{2}-8 \hat{\pi}_{1}(z)}}{4} \\ \frac{a+w_{2}}{2}, & \text { if } w_{2}<\frac{a+2 c}{3}\end{cases}
$$

When $\hat{\pi}_{1}(z) \geq \frac{(a-c)^{2}(1+4 \delta+\hat{p}(z) \cdot \rho)}{16(1+2 \delta+\hat{p}(z) \cdot \rho)}$, the supplier's optimal decision is

$$
w_{2}^{* *}= \begin{cases}\frac{a+c}{2}-\frac{\delta(a-c)}{2(1+2 \delta+\hat{p}(z) \cdot \rho)}, & \text { if } \delta<\frac{1+\hat{p}(z) \cdot \rho}{7} \\ \frac{a+2 c}{3}, & \text { otherwise }\end{cases}
$$

When $\hat{\pi}_{1}(z)<\frac{(a-c)^{2}(1+4 \delta+\hat{p}(z) \cdot \rho)}{16(1+2 \delta+\hat{p}(z) \cdot \rho)}$, the supplier's optimal decision is $w_{2}^{I I}$ if $\pi_{S, 2}\left(w_{2}^{I I}\right)>\pi_{S, 2}\left(\frac{a+2 c}{3}\right)$; otherwise, the optimal decision is $\frac{a+2 c}{3}$. 


\section{Best-Response Retail Price 4.}

The best-response retail price for the entire problem for $w_{2}^{I I} \leq w_{2}^{I}$ and $w_{2}^{I I I} \leq \frac{a(1+\delta)+c(2+\delta)}{3+2 \delta}$ is given as as follows

$$
p_{2}^{* *}= \begin{cases}\frac{a+w_{2}}{2}+\frac{\delta\left(w_{2}-c\right)}{2(1+\delta+\hat{p}(z) \cdot \rho)}, & \text { if } w_{2}<w_{2}^{I} \\ 2 w_{2}-c, & \text { if } \frac{a+2 c}{3} \leq w_{2} \leq w_{2}^{I} \\ \frac{a+w_{2}}{2}, & \text { if } w_{2}<\frac{a+2 c}{3}\end{cases}
$$

The supplier's optimal decision is

$$
w_{2}^{* *}= \begin{cases}\frac{a+c}{2}-\frac{\delta(a-c)}{2(1+2 \delta+\hat{p}(z) \cdot \rho)}, & \text { if } \delta<\frac{1+\hat{p}(z) \cdot \rho}{7} \\ \frac{a+2 c}{3}, & \text { otherwise }\end{cases}
$$

\subsection{Proofs}

\section{Proof of Lemma 1}

The lemma can be proved by direct comparison between the best response functions of Retailer 1 (in (B.1)) and Retailer 2 (in (B.8), (B.29), (B.32), (B.34) and (B.36)).

Lemma 3. Retailer's belief of Retailer 1's profit, $\hat{\pi}_{1}(z)$, is non-increasing in the wholesale price offer to Retailer 1, $w_{1}$.

Proof. Consider two wholesale prices $w_{1}$ and $w_{1}+\Delta$ for $\Delta>0$. For the same noise level $\epsilon$ and two signal realizations, $z=w_{1}+\epsilon$ and $z_{1}=w_{1}+\Delta+\epsilon$. In Retailer 2's inferences, the sets of possible wholesale price offers are respectively $[z-b, z+b]$ and $\left[z_{1}, z_{1}+b\right]$. The posterior probability densities are the same except that the one under $z_{1}$ is shifted to the right, i.e., $p(z+\kappa)=p\left(z_{1}+\kappa\right)$ for $\kappa=-b,-b+1, \ldots, b$. Suppose $z+\hat{\kappa}$ is the highest offer acceptable to Retailer 1 according Retailer 2's belief (i.e., $u_{1}(z+\hat{\kappa})>0$ and $\left.u_{1}(z+\hat{\kappa}+1) \leq 0\right)$. For small enough $\Delta>0, z_{1}+\hat{\kappa}$ is also the highest offer acceptable to Retailer 1 in Retailer 2's inference for the signal $z_{1}$. Therefore, according Retailer 2's belief, the acceptable offers for the signal $z$ and $z_{1}$ are $[z-b, z+\hat{\kappa}]$ and $\left[z_{1}-b, z_{1}+\hat{\kappa}\right]$, respectively. Note that according Lemma 2 , $\pi_{1}(z+\kappa) \geq \pi_{1}\left(z_{1}+\kappa\right)$ for $\kappa=-b,-b+1 \ldots, \hat{\kappa}$. Thus, the expected profits upon acceptance according to Retailer 2's inferences are such that $\hat{\pi}_{1}(z) \geq \hat{\pi}_{1}\left(z_{1}\right)$. In other words, $\hat{\pi}_{1}(z)$ is non-increasing in $w_{1}$.

Lemma 4. When the supplier is faced with two retailers and makes wholesale price offers sequentially, the optimal price offer to Retailer 1, $w_{1}^{* *}$, conditional upon Retailer 2's acceptance and choosing $p_{2}^{* *}$, is lower than the optimal wholesale price offer, $w_{1}^{*}=w_{i}^{*}$, made by the supplier when she makes these offers simultaneously (see Section 2.3).

Proof. Note that any wholesale price offer $w_{1}$ that is higher than $w_{1}^{*}$ in (2.3) reduces the supplier's profit from Retailer 1. Thus we only need to show that a higher price also lower the supplier's profit from Retailer 2. Note that $w_{1}$ affects the Retailer 2's response and consequently the supplier's profit 
through $\hat{p}(z)$ and $\hat{\pi}_{1}(z)$. Consider $w_{1}^{*}$ in eq. (2.3) and a wholesale price offer $w_{1}=w_{1}^{*}+\Delta$, and for the same noise $\epsilon$, the signal realizations are $z=w_{1}^{*}+\epsilon$ and $z_{1}=w_{1}^{*}+\Delta+\epsilon$. Note that $z_{1}=z+\Delta$, then in Retailer 2's inference, the set of possible wholesale prices under the actual wholesale price $w_{1}$ (i.e., $\left.\left[z_{1}-b, z_{1}+b\right]\right)$ is shifted to the right by $\Delta$ relative to the set of wholesale prices under the actual value of $w^{*}$ (i.e., $[z-b, z+b]$ ). Thus the probability of acceptance under $z_{1}$ can not be larger than that under $z$ because a higher wholesale price results in a lower profit and utility for Retailer 1 according to Lemma 2. Furthermore, by Lemma 3, the expected profit upon acceptance $\hat{\pi}_{1}\left(z_{1}\right)$ is no more than $\hat{\pi}_{1}(z)$. Therefore, for any common noise $\epsilon$ and a positive $\Delta$, a wholesale price $w_{1}=w^{*}+\Delta$ leads to $\hat{p}\left(z_{1}\right) \leq \hat{p}(z)$ and $\hat{\pi}_{1}\left(z_{1}\right) \leq \hat{\pi}_{1}(z)$. Furthermore, for any given $w_{2}$, the Retailer 2's best responses in eq. (B.8) and eq. (B.29), (B.32), (B.34) and (B.36) under the actual values of $w_{1}^{*}, w_{1}=w_{1}^{*}+\Delta$ and a common noise $\epsilon$, are such that $p_{2}^{*}\left(w_{2}, z_{1}\right) \geq p_{2}^{*}\left(w_{2}, z\right)$. As a result, the supplier's profits are $\left(a-p_{2}\left(w_{2}, z_{1}\right)\right)\left(w_{2}-c\right) \leq\left(a-p_{2}\left(w_{2}, z\right)\right)\left(w_{2}-c\right)$. Therefore, for each possible noise $\epsilon$, any wholesale price offer above $w_{1}^{*}$ results in a lower profit than $w_{1}^{*}$. The optimal wholesale price to Retailer 1 thus is not higher than $w_{1}^{*}$.

Lemma 5. When the supplier is faced with two retailers and makes wholesale price offers sequentially, the optimal wholesale price offer to Retailer 2, $w_{2}^{* *}$, conditional upon Retailer 2's acceptance and choosing $p_{2}^{* *}$, is higher than the optimal wholesale price offer, $w_{1}^{*}=w_{i}^{*}$, when the supplier makes these offers simultaneously (see Section 2.3).

Proof. Since the optimal wholesale price to Retailer 2 has closed-form expression, a direct comparison is sufficient to show that the optimal wholesale prices in Appendix 2.1 and 2.2 are all (weakly higher) than the one shown in (2.3).

\section{Proof of Proposition 1}

When only distributional fairness matters, Retailer $i$ 's best-response retail price in identical to eq. (B.1). Substituting (B.1) into the objective function $\pi_{S, i}=\left(a-p_{i}\right)\left(w_{i}-c\right)$, we have

$$
\pi_{S, i}= \begin{cases}\left(\frac{a-w_{i}}{2}-\frac{\delta\left(w_{i}-c\right)}{2(1+\delta)}\right)\left(w_{i}-c\right), & \text { if } w_{i} \geq \frac{a(1+\delta)+c(2+\delta)}{3+2 \delta} \\ \left(a-2 w_{i}+c\right)\left(w_{i}-c\right), & \frac{a+2 c}{3} \leq w_{i}<\frac{a(1+\delta)+c(2+\delta)}{3+2 \delta} \\ \frac{\left(a-w_{i}\right)\left(w_{i}-c\right)}{2}, & \text { if } w_{i}<\frac{a+2 c}{3}\end{cases}
$$

This objective function is piece-wise concave. The optimal solution can found by comparing the optimal values in each interval. Moreover, under the optimal wholesale price, the best-response retail price (B.1) always gives Retailer $i$ a positive utility and he always accepts.

\section{Proof of Proposition 2}

When $\rho=0$, the model reduces to the model with distributional fairness, and both retailers have positive utilities and accept the wholesale price offers as shown in the proof of Proposition 1 above. When $\rho>0$, 
Retailer 2's utility is negatively affected by $\rho \cdot \hat{p}(z)\left[\max \left\{\hat{\pi}_{1}(z)-\pi_{2}, 0\right\}\right]$. Suppose Retailer 2 chooses best-response retail price $p_{2}^{* *}$, then this best-response retail price can lead to a negative utility when $\rho$ is arbitrarily large. For the optimal wholesale price $w_{2}^{* *}$, the maximum value of peer-induced fairness parameter $\bar{\rho}$ with which Retailer 2 incurs a non-negative utility can be found by solving $u_{2}\left(p_{2}^{* *}\left(w_{2}^{* *}\right)\right)=0$ for $\rho$. When $\rho$ is not too large, i.e., $\rho<\bar{\rho}$, Retailer 2 accepts his wholesale price offer $w_{2}^{* *}$, and Retailer 1 accepts his wholesale price offer $w_{1}^{* *}$ because $w_{1}^{* *}$ is more attractive than the one without peer-induced fairness concern (by Lemma $4 w_{1}^{* *} \leq w_{i}^{*}$ ). The comparison between $w_{1}^{* *}$ and $w_{2}^{* *}$ follows directly by combining Lemmas 4 and 5 above.

\section{Proof of Proposition 3}

Following the proof of Proposition 2 above, when $\rho$ is not too large, i.e., $\rho<\bar{\rho}$, both retailers accept their wholesale price offers and choose best-response retailer prices. Since we have derived best-response retail prices in closed-from, the proof of this proposition follows direct comparisons of the retailers profits. 